TRANSACTIONS OF THE

AMERICAN MATHEMATICAL SOCIETY

Volume 275, Number 2, February 1983

\title{
SCHRÖDINGER OPERATORS WITH RAPIDLY OSCILLATING CENTRAL POTENTIALS ${ }^{1}$
}

\author{
BY
}

DENIS A. W. WHITE

\begin{abstract}
Spectral and scattering theory is discussed for the Schrödinger operators $H=-\Delta+V$ and $H_{0}=-\Delta$ when the potential $V$ is central and may be rapidly oscillating and unbounded. A spectral representation for $H$ is obtained along with the spectral properties of $H$. The existence and completeness of the modified wave operators is also demonstrated. Then a condition on $V$ is derived which is both necessary and sufficient for the Moller wave operators to exist and be complete. This last result disproves a recent conjecture of Mochizuki and Uchiyama.
\end{abstract}

1. Introduction. We shall study the Schrödinger operator $H=-\Delta+V$ on $L^{2}\left(\mathbf{R}^{d}\right)$ $(d \geqslant 2)$ when the potential $V$ is central, oscillating or perhaps rapidly oscillating, in which case $V$ may be unbounded. A spectral representation (or "eigenfunction expansion") for $H$ will be derived along with the "expected" spectral properties. We shall further prove the existence and completeness of the modified wave operators for the selfadjoint operators, $H$ and $H_{0}=-\Delta$ and derive a necessary and sufficient condition on $V$ to assure the existence and completeness of the Moller (i.e. "usual") wave operators.

To place this present work in perspective, we shall briefly review some of the relevant results from scattering theory. For potentials $V$ which are short range, i.e., roughly $V(x)=O\left(|x|^{-1-\varepsilon}\right)$ as $|x| \rightarrow \infty$, for some $\varepsilon>0$, Agmon [1] has shown that the Moller wave operators exist and are complete. (For a complete bibliography before 1978, see [44].) More recently, Enss [21] has obtained a similar result by an elegant time dependent method; see also $[23,44,48,53]$. On the other hand, Dollard $[17,18]$ has shown that these wave operators do not exist when $V$ is the Coulomb potential, $V(x)=r^{-1}$ (where $r=|x|$ ). It is not true, however, that these wave operators fail to exist for all potentials which are not short range. Consider, for example

$$
V(x)=r^{-\delta} \sin \beta r^{\alpha} \quad(\alpha \geqslant 0 ; \delta>\min (0,1-\alpha) ; \beta>0)
$$

(so that $V$ depends only on $r=|x|$, i.e. $V$ is central), which is typical of the potentials discussed below. These potentials have been the object of study in several recent papers $[11,12,16,19,37,38,52,54]$ and they are special cases of more general works about oscillating potentials $[3,14,15,35,36,41,47]$, see also [6]. These authors find certain conditions on $\delta$ and $\alpha$ in (1.1) weaker than $\delta>1$ (i.e. the

Received by the editors July 20, 1981 and, in revised form, December 22, 1981.

1980 Mathematics Subject Classification. Primary 35P25; Secondary 35P10.

'Research supported in part by a N.A.T.O. postdoctoral fellowship awarded by N.S.E.R.C. (Canada). 
short range case) which are still sufficient to assure the existence and completeness of the Moller wave operators. We cite but a few: $\delta+\alpha>2[15,47] ; \frac{3}{2}<\delta+\alpha<2$ and $\delta+2 \alpha>3[14,41] ; \delta+\alpha>1$ and $\delta>\frac{1}{2}$ [16]; $2 \delta+\alpha>2$ and $\delta>0$ [11]. (In each case we assume $V$ is not too singular at 0 , say $\alpha-\delta>-2$.)

Faced with this bewildering number of sufficient conditions it is natural to ask if any is necessary. Bourgeois [12] conjectured that if $\alpha=1$, then $\delta>\frac{1}{2}$ is necessary. See also [54]. A much more general conjecture is the following, due to Mochizuki and Uchiyama [39]: if $V$ is continuous and central the Moller wave operators exist if $V$ is conditionally integrable on $(1, \infty)$ (i.e. $\int_{1}^{\infty} V(s) d s$ exists as an improper Riemann integral). For the potentials in (1.1), conditional integrability is equivalent to $\delta+\alpha>1$ so that the two conjectures are incompatible. In this paper we shall resolve these conflicting conjectures. More precisely, we shall restrict attention to a certain class of central potentials including those in (1.1) and derive a necessary and sufficient condition on $V$ in this class for Møller wave operators to exist and be complete. Thus we shall prove that Mochizuki and Uchiyama's condition is not sufficient but is necessary at least in this context. Bourgeois's conjecture is correct.

The procedure here is to prove the existence and completeness of the modified wave operators and to derive our results about Moller wave operators from there. The modified wave operators are of interest in their own right and have been studied extensively $[22,26,27,32,34,42,45]$. However, these results do not apply to rapidly oscillating potentials. For example, Hörmander's existence result [26] requires $\delta>3 \alpha$ for the potentials in (1.1). To accommodate oscillating potentials, we shall use the method of Green and Lanford [24] to prove the existence of the wave operators, and not Cook's method which was used in the previously mentioned works. The former method is the one applied by Dollard and Friedman [19] who were the first to treat the important case (among others) when $V(r)=r^{-1} \sin r$.

To apply the Green-Lanford method, we shall require a spectral representation for $H$. Because such representations are important in any analysis of the operator $H$, we shall derive it in more generality than is strictly required for considering the wave operators. Thus we shall prove that $H_{0}$ and the absolutely continuous part of $H$ are unitarily equivalent, for all $V$ in (1.1). This generalizes Ben-Artzi's results [8,9] as they apply to the potentials in (1.1); he required $0 \leqslant \alpha<1$. However we do not recover his partial results in the case $\delta=0,0 \leqslant \alpha<1$. The "usual" spectral properties of $H$ will also be verified; see Theorem 1.1 below. For other spectral representations for $H$ when $V$ is not necessarily short range, see [4, 7, 28, 37, 38, 49, 51], for example. Their results do not apply to all the potentials in (1.1).

We now give complete statements of our hypotheses and results. Some notation is required.

Notation. Fix $\alpha \geqslant 0$ and $\beta>0$. For each of $\delta_{1} \in \mathbf{R}$ and $\delta_{2}>0$ define $C\left(\delta_{1}, \delta_{2}\right)$ to be the set of all infinitely differentiable functions $f: \mathbf{R}_{+} \rightarrow \mathbf{C}\left(\mathbf{R}_{+}=(0, \infty)\right)$ such that, for all $m \in \mathbf{N}(\mathbf{N}=\{0,1,2, \ldots\})$

$$
f^{(m)}(r)=O\left(r^{-\delta_{1}-m \delta_{2}}\right) \quad \text { as } r \rightarrow \infty .
$$


Define $B\left(\delta_{1}, \delta_{2}\right)$ to be the set of all real-valued functions $V_{L}$ of the form

$$
V_{L}(r)=g_{0}(r)+\sum_{k=1}^{n} g_{k}(r) \cos k \beta r^{\alpha}+f_{k}(r) \sin k \beta r^{\alpha} \quad(r>0)
$$

where each $g_{k}(0 \leqslant k \leqslant n)$ and $f_{k}(1 \leqslant k \leqslant n)$ belong to $C\left(\delta_{1}, \delta_{2}\right)$ and is real-valued, and $n \in \mathbf{N}$. If $g_{0}=0$, then $V_{L}$ is said to be oscillatory.

Hypotheses. We shall assume that $V: \mathbf{R}_{+} \rightarrow \mathbf{R}$ satisfies

(V1) $V \in L_{\text {loc }}^{2}\left(\mathbf{R}_{+}\right)$.

(V2) $V(r)=O\left(r^{-2+\varepsilon}\right)$ as $r \rightarrow 0$, for some $\varepsilon>0$.

$\left(V 2^{\prime}\right) \int_{0}^{1}(V(r))^{2} r^{d-1} d r<\infty$.

(V3) $V=V_{L}+V_{S}=V_{L 1}+V_{L 2}+V_{S}$ where, for some $\delta_{1}>0$ and $\delta_{2}, 0<\delta_{2} \leqslant 1$,

$(S R) V_{S}(r)=O\left(r^{-2-\varepsilon}\right)$ as $r \rightarrow \infty(\varepsilon>0)$ and $V_{S}$ is real-valued and continuous on $\left(r_{0}, \infty\right)$ for some $r_{0}>0$.

(LR1) $V_{L 1} \in B\left(\delta_{1}, \delta_{2}\right)$.

(LR2) $V_{L 2}=0$ if $\alpha \leqslant 1$ but if $\alpha>1$, then $V_{L 2} \in B\left(\delta_{1}-(\alpha-1), \delta_{2}\right)$ and $V_{L 2}$ is oscillatory.

Observe that $(V 2)$ implies $\left(V 2^{\prime}\right)$ when $d \geqslant 4$ (we are working in $\mathbf{R}^{d}, d \geqslant 2$ ) and that the assumption $\delta_{2} \leqslant 1$ in $(V 3)$ does not restrict generality. Also, given that $V_{L}=V_{L 1}+V_{L 2}$ belongs to $B\left(\delta, \delta_{2}\right)(\delta \in \mathbf{R}),(L R 1)$ and $(L R 2)$ say essentially that $V_{L 1}(r) \rightarrow 0$ as $r \rightarrow \infty$ and $V_{L 2}$ is conditionally integrable on $(1, \infty)$.

The choice of the function $\beta r^{\alpha}$ in (1.3) is made for convenience and to include the important case $\alpha=1$. It could be replaced by $f$ where either $f^{\prime}$ or $1 / f^{\prime}$ belongs to $C\left(\delta, \delta_{2}\right)(\delta>0)$ (corresponding to the cases $\alpha<1$ or $\alpha>1$ here). Thus the results below apply to $V_{L}(r)=V_{L 2}(r)=r^{-\delta_{1}} e^{r} \sin e^{r}\left(\delta_{1}>0\right)$ for example.

The assumption $(S R)$ is not the best possible (see [16]) but we are interested here primarily in $V_{L}$ and include $V_{S}$ only to weaken the smoothness assumption on $V$.

Of course $V=V_{L}$ defined by (1.1) satisfies (V1) and either ( $\left.L R 1\right)$ or ( $\left.L R 2\right)$, but additional restrictions on $\delta$ and $\alpha$ are required if $V$ is to satisfy $(V 2)$ and $\left(V 2^{\prime}\right)$.

Results. With the above assumptions we shall obtain a spectral representation (Theorem 8.2) for the absolutely continuous part of $H=-\Delta+V$ from which we shall derive the following result about the spectral properties of $H$.

THEOREM 1.1. Suppose $V$ satisfies $(V 1),(V 2),\left(V 2^{\prime}\right)$ and $(V 3)$ and set $H=-\Delta+V$. Then the spectrum of $H$ is bounded below and its singularly continuous spectrum is void. If $\alpha \neq 1$ then $H$ has no (strictly) positive eigenvalues, but if $\alpha=1$ then $H$ may have at most finitely many positive eigenvalues and they must belong to the set $\left\{\frac{1}{4}(k \beta)^{2}\right.$ : $k \in N\}$. Finally, the absolutely continuous part of $H$ is unitarily equivalent to $H_{0}=-\Delta$.

In the case $V(r) \rightarrow 0$ as $r \rightarrow \infty$ the conclusions of Theorem 1.1 may be slightly strengthened; see Remark 9.1. The existence of positive eigenvalues when $\alpha=1$ is indeed possible; see $[35,40,49,50]$.

The next two results concern the existence of the wave operators beginning with the modified wave operators. We do not indicate here just how the modified wave operators should be modified, but this will be clarified in the text (see (10.1) and (10.2)). 
THEOREM 1.2. Suppose $V$ satisfies $(V 1),(V 2),\left(V 2^{\prime}\right)$, and $(V 3)$, and let $V_{L 1}, \delta_{1}$, and $\delta_{2}$ be as in assumption (V3). Denote by $H$ and $H_{0}$ the selfadjoint operators on $L^{2}\left(\mathbf{R}^{d}\right)$ $(d \geqslant 2), H=-\Delta+V$ and $H_{0}=-\Delta$. If $\delta_{2}>\frac{1}{2}$ and at least one of the conditions:

(a) $\delta_{1}>\frac{1}{2}$;

(b) $\delta_{1}>\frac{1}{4}, V_{L 1}$ is conditionally integrable on $(1, \infty)$ and for some $\varepsilon>0$

$$
\int_{r}^{\infty} V_{L 1}(\sigma) d \sigma=O\left(r^{-\varepsilon}\right) \text { as } r \rightarrow \infty
$$

is satisfied, then the modified wave operators for $H$ and $H_{0}$ exist and are complete.

Combining this result with a result of Hörmander's (Lemma 10.1 below) it is possible to obtain a necessary and sufficient condition for the existence and completeness of the Moller wave operators. For the sake of simplicity we state this result only in a special case, the case when, for $r>0$,

$$
V_{L}(r)=a r^{-\delta} \sin \beta r^{\alpha} \quad(a \in \mathbf{R} ; \beta>0 ; \alpha \geqslant 0 ; \delta>\min (0,1-\alpha)) .
$$

TheOrem 1.3. Let $V_{L}, \delta$, and $\alpha$ be as in (1.4). Suppose that $\delta$ and $\alpha$ satisfy at least one of the following conditions:

(i) $\delta>\frac{1}{2}$;

(ii) $\delta>\frac{1}{4}$ and $\delta+\alpha>1$;

(iii) $\delta+\alpha>\frac{5}{4}$.

Suppose further that $V_{S}: \mathbf{R}_{+} \rightarrow \mathbf{R}$ satisfies $(S R)$ and $V=V_{L}+V_{S}$ satisfies $(V 1),(V 2)$, and $\left(V 2^{\prime}\right)$. Denote by $H$ and $H_{0}$ the selfadjoint operators on $L^{2}\left(\mathbf{R}^{d}\right)(d \geqslant 2), H=-\Delta$ $+V$ and $H_{0}=-\Delta$. Then the modified wave operators for $H$ and $H_{0}$ exist and are complete. The Moller wave operators for $H$ and $H_{0}$ exist if and only if either $V_{L}=0$ or $\delta$ and $\alpha$ satisfy at least one of the following conditions:

(iv) $\delta+\alpha>1$ and $\delta>\frac{1}{2}$;

(v) $\delta+\alpha>\frac{3}{2}$.

In this event, the Moller wave operators are complete.

The conjectures of Mochizuki and Uchiyama and of Bourgeois are resolved by Theorem 1.3; see Remark 12.1 below.

The plan of this paper is as follows. In $\$ 2$ we decompose $H$ as a direct sum of ordinary differential operators, $H(l)$. We examine the asymptotic behavior of the generalized eigenfunctions of $H(l)$ in $\$ \S 3-6$; this constitutes the bulk of the technical work of this paper. In $\S 7$, the spectral properties of $H(l)$ are derived by way of a limiting absorption principle. The spectral representation theorem and Theorem 1.1 are established in $\$ \S 8$ and 9 . In $\$ 10$ we introduce the modified and Møller wave operators, and establish Theorems 1.2 and 1.3 in $\$ \S 11$ and 12.

2. Preliminaries. In this section we establish two results which will simplify the analysis in the rest of the paper. The first simplification involves reformulating hypothesis $(V 3)$ in a more convenient form. 
LEMMA 2.1. Let $V_{L}, \delta_{1}>0$ and $\delta_{2}, 0<\delta_{2} \leqslant 1$, be as in assumption (V3). Then $V_{L}=V_{L 1}+V_{L 2}$ where

( $L R 1) V_{L 1} \in B\left(\delta_{1}, \delta_{2}\right)$;

(LR2') if $\alpha \leqslant 1$, then $V_{L 2}=0$, but if $\alpha>1$, then $V_{L 2}$ in $B\left(\delta_{1}-(\alpha-1), \delta_{2}\right)$ is oscillatory and $\int_{1}^{\infty} V_{L 2}(s) d s$ exists conditionally and the function $r \mapsto \int_{r}^{\infty} V_{L 2}(s) d s$ belongs to $B\left(\delta_{1}, \delta_{2}\right)$ and is oscillatory.

Proof. Let $W_{1}$ and $W_{2}$ be the functions denoted by $V_{L 1}$ and $V_{L 2}$ in assumption (V3). Assume that $\alpha>1$ (otherwise the result is trivial) and that $W_{2}(r)=$ $f(r) \cos k \beta r^{\alpha}$ for some $f \in C\left(\delta_{1}-(\alpha-1), \delta_{2}\right)$, and $k \in \mathbf{Z}$ where $k \neq 0$ (i.e., $W_{2}$ is oscillatory). Integrate by parts

$$
\begin{aligned}
\int_{r}^{\infty} W_{2}(s) d s= & -(k \beta \alpha)^{-1} r^{1-\alpha} f(r) \sin k \beta r^{\alpha} \\
& -(k \beta \alpha)^{-1} \int_{r}^{\infty} \frac{d}{d s}\left(s^{1-\alpha} f(s)\right) \sin k \beta s^{\alpha} d s .
\end{aligned}
$$

Here we have used the fact that $r^{1-\alpha} f(r)$ belongs to $C\left(\delta_{1}, \delta_{2}\right)\left(\delta_{1}>0\right)$ to eliminate the boundary term at $\infty$. Therefore setting

$$
V_{L 2}(r)=W_{2}(r)+(k \beta \alpha)^{-1}\left(\frac{d}{d r}\right)\left(r^{1-\alpha} f(r)\right) \sin k \beta r^{\alpha}
$$

and $V_{L 1}=V_{L}-V_{L 2}$ the result follows in the case $W_{2}(r)=f(r) \cos k \beta r^{\alpha}$. A similar argument applies when $W_{2}(r)=f(r) \sin k \beta r^{\alpha}$. In general, $W_{2}$ is a finite sum of such functions and hence the result follows.

The second simplification entails rewriting $H=-\Delta+V$ as a direct sum of selfadjoint ordinary differential operators. Begin by recalling the definition of $H$ and $H_{0}=-\Delta$. Suppose $V$ satisfies $(V 1),(V 2),\left(V 2^{\prime}\right)$ and $(V 3)$. For $f \in C_{0}^{\infty}\left(\mathbf{R}^{d}\right)$ (the space of infinitely differentiable functions from $\mathbf{R}^{d}$ to $\mathbf{C}$ with compact support), the expressions

$$
H_{0}^{\prime} f(x)=-\Delta f(x), \quad H^{\prime} f(x)=-\Delta f(x)+V(|x|) f(x)
$$

make sense and define operators $H_{0}^{\prime}$ and $H^{\prime}$ on $L^{2}\left(\mathbf{R}^{d}\right)$. The operator $H_{0}^{\prime}$ is essentially selfadjoint [31, p. 299]; we shall refer to its closure by $H_{0}=$ " $-\Delta$ " in future. The operator $H^{\prime}$ certainly has a selfadjoint extension [20, Corollary 13, p. 1230]; we choose one and refer to it by $H=$ “ $-\Delta+V$ ". Later (in Lemma 7.2) we shall see that $H^{\prime}$ is essentially selfadjoint so that $H$ is uniquely determined.

We recall that the operator $H=$ " $-\Delta+V$ " can be decomposed into selfadjoint ordinary differential operators defined on $L^{2}\left(\mathbf{R}_{+}\right)$; this is possible because $V$ is central. More precisely, there is a decomposition of $L^{2}\left(\mathbf{R}^{d}\right)$,

$$
L^{2}\left(\mathbf{R}^{d}\right)=\bigoplus_{l \in \mathbf{N}} \mathcal{H}_{l}
$$

into closed, mutually orthogonal subspaces, $\mathcal{H}_{l}$ such that for each $l \in \mathbf{N}$ there is a unitary operator $U_{l}$ from $L^{2}\left(\mathbf{R}_{+}\right)$onto $\mathcal{H}_{l}$. Moreover, for any $V$ satisfying $(V 1)$, $(V 2),\left(V 2^{\prime}\right)$, and $(V 3)$, if $H={ }^{\prime \prime}-\Delta+V "$, then each of the subspaces reduces $H$ (i.e. 
$P_{l} H \subseteq H P_{l}$ if $P_{l}$ is the corresponding projection operator) and $H(l)$ defined by $H(l)=U_{l}^{-1} H U_{l}$ is a selfadjoint operator associated with the differential expression

$$
\tau_{l}=-\frac{d^{2}}{d r^{2}}+\left(\nu(l) r^{-2}+V(r)\right) \quad(r>0)
$$

where $\nu(l)$ is some constant, $\nu(l) \geqslant-\frac{1}{4}$. Finally, if

$$
D\left(T_{1}\left(\tau_{l}\right)\right)=\left\{f \in L^{2}\left(\mathbf{R}_{+}\right): \tau_{l} f \in L^{2}\left(\mathbf{R}_{+}\right)\right\}
$$

(when we write $\tau_{l} f$ it is understood that $f$ has absolutely continuous first derivative) then the domain, $D(H(l))$ of $H(l)$ where

$$
D(H(l)) \subseteq\left\{f \in D\left(T_{1}\left(\tau_{l}\right)\right): B_{l} f=0\right\},
$$

where

$$
B_{l}(f)= \begin{cases}\lim _{r \rightarrow 0} r^{1 / 2}(\log r)^{-1} f^{\prime}(r) & \text { if } \nu(l)=-\frac{1}{4}, \\ \lim _{r \rightarrow 0} f(r) & \text { if } \nu(l)=0, \\ 0 & \text { otherwise. }\end{cases}
$$

For a proof of the result, see [30, 43 or 11 , Appendix].

In future $H(l)$ will be referred to as the operator defined in $\S 2$. The analogous operator, corresponding to $H_{0}=$ " $-\Delta$ ", will be denoted $H_{0}(l)$. Much of the rest of this paper is devoted to studying the spectral and scattering theoretical properties of $H(l)$ and $H_{0}(l)$. Using the above result we shall derive the comparable properties for $H$ and $H_{0}$.

3. Asymptotic behavior. We have reduced the study of the operators $H$ and $H_{0}$ on $L^{2}\left(\mathbf{R}^{d}\right)$ to the study of the operators $H(l)$ and $H_{0}(l)$ on $L^{2}\left(\mathbf{R}_{+}\right)$. We begin our analysis of $H(l)$ by considering the asymptotic behavior at 0 and at $\infty$ of solutions of the equation $\left(\tau_{l}-z^{2}\right) \sigma=0$, i.e., of the generalized eigenfunctions of $H(l)\left(\tau_{l}\right.$ defined by (2.1)). Knowledge of this asymptotic behavior is fundamental to determining the spectral properties of $H(l)$ and hence of $H$. The first result concerns this behavior at 0 .

LEMma 3.1. Let $\tau_{l}$ be, for some $l \in \mathbf{N}$, the differential expression defined by (2.1) and suppose $V$ there satisfies $(V 1)$ and $(V 2)$. Then there is a function $\psi: \mathbf{R}_{+} \times \mathbf{C} \rightarrow \mathbf{C}$ which satisfies:
(a) $\left(\tau_{l}-z^{2}\right) \psi(\cdot, z)=0$;
(b) $\psi$ and $\psi^{\prime}$ are continuous;
(c) the functions $z \rightarrow \psi(r, z)$ and $z \rightarrow \psi^{\prime}(r, z)$ are, for each fixed $r$, holomorphic on C;

(d) $\overline{\psi(r, \bar{z})}=\psi(r, z)=\psi(r,-z)$ for all $(r, z) \in \mathbf{R}_{+} \times \mathbf{C}$;

(e) $\psi \in L^{2}((0,1) \times \Lambda)$ for any compact interval $\Lambda$ in $\mathbf{R}$;

(f) $B, \psi(\cdot, z)=0$ for every $z \in \mathbf{C}\left(B_{l}\right.$ was defined in (2.3));

(g) for each $z \in \mathbf{C}, \psi(\cdot, z)$ has only finitely many zeros in $(0,1]$.

Finally, any solution of (a) in $L^{2}((0,1))$ satisfying (f) is a multiple of $\psi(\cdot, z)$. 
For a proof of this result see [5, Lemmas 3.3 and A.1; 2 or 43].

We shall next consider the asymptotic behavior at $\infty$ of solutions of the equation $\left(\tau-z^{2}\right) \sigma=0$ where

$$
\tau=-\frac{d^{2}}{d r^{2}}+V(r) \quad(r>0) .
$$

Of primary interest is the case when $V$ is as in assumption ( $V 3)$, but it is convenient to allow more general choices of $V$.

The estimates of the asymptotic behavior will be obtained by repeated integration by parts. In order to handle the resultant cumbersome expressions, we shall need an integration by parts "machine". The set of functions $E$ introduced below will serve this purpose.

Notation. Recall the fixed constants, $\alpha \geqslant 0$ and $\beta>0$ of $\S 1$. Define the following subsets of the complex plane, $\mathbf{C}$. For each $j \in \mathbf{N}$, let

$$
\mathbf{C}_{j}=\mathbf{C}-\left\{\frac{1}{2} k \beta: 0 \leqslant|k| \leqslant j\right\}
$$

and

$$
\mathbf{C}_{j}^{+}=\mathbf{C}_{j} \cap\{z \in \mathbf{C}: \operatorname{Im} z \geqslant 0\} .
$$

Here and below $\operatorname{Im} z$ and $\operatorname{Re} z$ denote the imaginary and real parts of $z$.

Define the set $D$. If $\alpha \neq 1$, then $D$ is the set of all functions $h$ holomorphic on $\mathbf{C}_{0}$. If $\alpha=1$, then $D$ is the set of all functions holomorphic on $\mathbf{C}_{j}$, for some $j \in \mathbf{N}$ depending on $h$. The somewhat different definition of $D$ when $\alpha=1$ is warranted because, in this case, a form of resonance occurs in the equation $\left(\tau_{l}-z^{2}\right) \sigma=0$, if $z=\frac{1}{2} k \beta$. As we shall see in Corollary 7.6, these values of $z^{2}$ may be eigenvalues of $H(l)$ and hence of $H$ and therefore must be excluded from consideration.

Let $\delta_{1} \in \mathbf{R}$ and $\delta_{2}>0$ be arbitrary. Recall, from $\S 1$, how $C\left(\delta_{1}, \delta_{2}\right)$ was defined. Define $E_{0}\left(\delta_{1}, \delta_{2}\right)$ to be the set of all functions which are finite sums of functions of the form

$$
f(r, z)=g(r) h(z) \text { for some } g \in C\left(\delta_{1}, \delta_{2}\right) \text { and } h \in D .
$$

Define $E\left(\delta_{1}, \delta_{2}\right)$ to be the set of all functions $f$ of the form

$$
f(r, z)=\sum_{k=-n}^{n} e^{i k \beta r^{\alpha}} f_{k}(r, z)
$$

for some set $\left\{f_{k}\right\}_{k=-n}^{n}$ in $E_{0}\left(\delta_{1}, \delta_{2}\right)$. The function $f$ will be said to be oscillatory if $f_{0}=0$. Define

$$
E=\bigcup\left\{E\left(\delta_{1}, \delta_{2}\right): \delta_{1} \in \mathbf{R}, \delta_{2}>0\right\} .
$$

We observe that a function $f \in E\left(\delta_{1}, \delta_{2}\right)$ has domain $\mathbf{R}_{+} \times \mathbf{C}_{j}$ for some $j \in \mathbf{N}$ and that $j=0$ if $\alpha \neq 1$. Moreover, by (1.2), if $K \subseteq \mathbf{C}_{j}$ is compact, then there is $C>0$ so that

$$
|f(r, z)|<C r^{-\delta_{1}} \text { for all }(r, z) \in(1, \infty) \times K .
$$

Thus, the set $E$ can be used to record asymptotic behavior. 
If $f \in E$, then we shall denote by $\bar{f}$ and $f_{-}$the functions defined by

$$
\begin{aligned}
\bar{f}(r, z) & =\overline{f(r, \bar{z})}, \\
f_{-}(r, z) & =f(r,-z) .
\end{aligned}
$$

We record for future reference the algebraic properties of the set $E$.

LEMMA 3.2. If $\boldsymbol{\delta}_{1} \in \mathbf{R}, \varepsilon_{1} \in \mathbf{R}, \boldsymbol{\delta}_{2}>0$ and $\varepsilon_{2}>0$, then:

(i) $E\left(\delta_{1}, \delta_{2}\right)$ is a linear space over $\mathrm{C}$;

(ii) if $f \in E\left(\delta_{1}, \delta_{2}\right)$ and $g \in E\left(\varepsilon_{1}, \varepsilon_{2}\right)$, then $f g \in E\left(\delta_{1}+\varepsilon_{1}, \min \left(\delta_{2}, \varepsilon_{2}\right)\right)$;

(iii) if $\varepsilon_{1} \leqslant \delta_{1}$ and $\varepsilon_{2} \leqslant \delta_{2}$, then $E\left(\delta_{1}, \delta_{2}\right) \subseteq E\left(\varepsilon_{1}, \varepsilon_{2}\right)$;

(iv) if $f \in E\left(\delta_{1}, \delta_{2}\right)$, then $\bar{f}$ and $f_{-}$also belong to $E\left(\delta_{1}, \delta_{2}\right)$;

(v) if $f \in E\left(\delta_{1}, \delta_{2}\right)$, then $d f / d z \in E\left(\delta_{1}, \delta_{2}\right)$.

Properties (i) through (v) remain true if " $E$ " is replaced throughout by " $E_{0}$ ". Moreover,

(vi) if $f \in E_{0}\left(\delta_{1}, \delta_{2}\right)$, then $f^{\prime} \in E_{0}\left(\delta_{1}+\delta_{2}, \delta_{2}\right)$.

Here "prime" denotes differentiation with respect to the first (real) variable where " $d / d z$ " denotes differentiation with respect to the second (complex) variable.

Proof. It is clear from the definitions that $E\left(\delta_{1}, \delta_{2}\right)$ and $E_{0}\left(\delta_{1}, \delta_{2}\right)$ are linear spaces over $\mathbf{C}$ (i.e., property (i) is verified). Therefore, it is enough to prove the remaining statements for $f$ in $E$ (respectively in $E_{0}$ ) of the form

$$
f(r, z)=e^{i k \beta r^{\alpha}} g(r) h(z) \quad \text { where } k \in \mathbf{Z}, g \in C\left(\delta_{1}, \delta_{2}\right), h \in D
$$

(respectively, $f=g h$ ). The verification in this simplified case is left to the reader.

Recall the set $B\left(\delta_{1}, \delta_{2}\right)$ of functions introduced in $\S 1$. It may be considered to be a subset of $E\left(\delta_{1}, \delta_{2}\right)$ because $g \in B\left(\delta_{1}, \delta_{2}\right)$ can be regarded as a function $g: \mathbf{R}_{+} \times \mathbf{C}_{0}$ $\rightarrow \mathbf{R}$ which does not depend on the second variable and because $\cos k \beta r^{\alpha}$ and $\sin k \beta r^{\alpha}$ can be written in terms of the exponentials $e^{i k \beta r^{\alpha}}$ and $e^{-i k \beta r^{\alpha}}$. Hence, $B\left(\delta_{1}, \delta_{2}\right) \subseteq E\left(\delta_{1}, \delta_{2}\right)$. Clearly the two definitions of oscillatory coincide on $B\left(\delta_{1}, \delta_{2}\right)$.

One final definition will complete the structure that we shall need for the consideration of asymptotic behavior. Let $A$ be the set of all functions which are finite sums of functions $f$ of the form $f(r, z)=g(r) h(z)$ for some $h \in D$ and some continuous function $g: \mathbf{R}_{+} \rightarrow \mathbf{C}$ such that $g(r)=O\left(r^{-\delta}\right)$ as $r \rightarrow \infty$ for some $\delta>0$ ( $\delta$ depending on $g$ ). It is clear that $A$ is an algebra of functions and $E\left(\delta_{1}, \delta_{2}\right) \subseteq A$ if $\delta_{1}>0$ and $\delta_{2}>0$.

Return now to the consideration of the asymptotic behavior of solutions of $\left(\tau-z^{2}\right) \sigma=0(\tau$ defined by (3.1)). The desired asymptotic behavior is introduced as a property of $V$. Suppose $V \in L_{\text {loc }}^{2}\left(\mathbf{R}_{+}\right)$. Then $V$ will be said to satisfy $\operatorname{Cond}(\xi)$ for some $\xi \in E$ if

$\operatorname{Cond}(\xi)$ : The function $\xi$ satisfies $\xi=\bar{\xi}=\xi_{-}$. Moreover, for some $j \in \mathbf{N}$ (and $j=0$ if $\boldsymbol{\alpha} \neq 1$ ) there exists $\phi: \mathbf{R}_{+} \times \mathbf{C}_{j}^{+} \rightarrow \mathbf{C}$ such that:

(1) $\left(\tau-z^{2}\right) \phi(\cdot, z)=0$ for all $z \in \mathbf{C}_{j}^{+}(\tau$ defined by $(3.1))$;

(2) $\phi$ and $\phi^{\prime}$ are continuous;

(3) the functions $\lambda \rightarrow \phi(r, \lambda)$ and $\lambda \rightarrow \phi^{\prime}(r, \lambda)$ are, for each fixed $r$, continuously differentiable on $\mathbf{C}_{j}^{+} \cap \mathbf{R}_{+}$; 


$$
\begin{aligned}
& \phi(r, z)=\exp \left\{i z\left(r+\int_{1}^{r} \xi(\sigma, z) d \sigma\right)\right\}\left(1+\eta_{1}(r, z)+\eta_{2}(r, z)\right), \\
& \phi^{\prime}(r, z)=i z \exp \left\{i z\left(r+\int_{1}^{r} \xi(\sigma, z) d \sigma\right)\right\}\left(1+\eta_{3}(r, z)\right)
\end{aligned}
$$

for all $(r, z) \in \mathbf{R}_{+} \times \mathbf{C}_{j}^{+}$. Here $\eta_{1} \in A$ and $\eta_{2}$ and $\eta_{3}$ are continuous functions of $\mathbf{R}_{+} \times \mathbf{C}_{j}^{+}$onto $\mathbf{C}$ such that $\eta_{2} \in L^{2}((1, \infty) \times \Lambda)$ for every compact subset $\Lambda$ of $\mathbf{C}_{j}^{+} \cap \mathbf{R}_{+}$and for each $z \in \mathbf{C}_{j}^{+}$

$$
\left|\eta_{2}(r, z)\right|+\left|\eta_{3}(r, z)\right|=o(1) \text { as } r \rightarrow \infty \text {. }
$$

The function $\phi$ of $\operatorname{Cond}(\xi)$ is (for an appropriate choice of $V$ ) a generalized eigenfunction of $H(l)$ and the function $\int_{1}^{r} \xi(\sigma, z) d \sigma$ is its generalized phase shift. In Theorem 8.2 below, we shall derive a spectral representation for the absolutely continuous part of $H(l)$ which will be an integral operator with kernel which may be expressed in terms of $\phi$. Thus, the above properties of $\phi$ combined with an estimate of $\xi$ to be derived in Lemma 6.1 below will provide information about this kernel which will enhance the usefulness of the representation.

The next result shows how it is sometimes possible to simplify the function $\xi$ of $\operatorname{Cond}(\xi)$.

Lemma 3.3. Suppose $V \in L_{\text {loc }}^{2}\left(\mathbf{R}_{+}\right)$satisfies $\operatorname{Cond}(\xi)$ for some $\xi \in E$. Suppose further that $\xi=\xi_{1}+\xi_{2}$ where $\xi_{1} \in E, \bar{\xi}_{1}=\xi_{1}=\left(\xi_{1}\right)_{-}$and that $\int_{1}^{\infty} \xi_{2}(s, z) d s$ exists conditionally. If $\zeta$ defined by $\zeta(r, z)=\int_{r}^{\infty} \xi_{2}(s, z) d s$ belongs to $A$ then $V$ satisfies $\operatorname{Cond}\left(\xi_{1}\right)$.

Proof. Let $\phi$ be the function appearing in $\operatorname{Cond}(\xi)$. Define

$$
\phi_{1}(r, z)=e^{-i z \zeta(1, z)} \phi(r, z)
$$

If it is shown that (1), (2), (3), and (4) of Cond $(\xi)$ hold when $\phi$ there is replaced by $\phi_{1}$ and $\xi$ by $\xi_{1}$, then the result will follow. Certainly $\phi_{1}$ satisfies (1), (2), and (3). To verify (4), observe that the function $e^{-i z \xi(r, z)}\left(1+\eta_{1}(r, z)+\eta_{2}(r, z)\right)$ can be written as $1+\rho_{1}+\rho_{2}$ for some functions $\rho_{1}$ and $\rho_{2}$ which have the same properties as do $\eta_{1}$ and $\eta_{2}$. This can be shown by expanding the exponential in its Maclaurin series. Similarly, $e^{-i z \zeta(r, z)}\left(1+\eta_{3}(r, z)\right)$ is of the same form as $1+\eta_{3}$. The result now follows.

We shall now state our main result about the asymptotic behavior at $\infty$ of solutions of $\left(\tau-z^{2}\right)=0(\tau$ defined by (3.1)).

THEOREM 3.4. Suppose $V$ satisfies $(V 1)$ and $(V 3)$ and let $\delta_{1}$ and $\delta_{2}$ be the constants there. Then there exists $\xi \in E\left(\delta_{1}, \delta_{2}\right)$ so that, for every $\nu \in \mathbf{R}$, the functions $r \mapsto \nu r^{-2}$ $+V(r)$ satisfy $\operatorname{Cond}(\xi)$. Moreover, the corresponding functions $\phi\left(=\phi_{\nu}\right)$ in $\operatorname{Cond}(\xi)$ are all defined on $\mathbf{R}_{+} \times \mathbf{C}_{j}^{+}$for some $j \in \mathbf{N}$ which does not depend on $\nu$.

Its and Matveev [29] have announced a similar result obtained by different methods. Theorem 3.4 may follow by their techniques. See also [5, 11] for related results. We establish Theorem 3.4 in the next three sections. 
4. Proof of Theorem 3.4. We turn now to the proof of Theorem 3.4. For the sake of simplicity, only the case $\nu=0$ is considered; the general case follows by a minor alteration to the proof of Lemma 4.1 below; see Remark 6.4.

Recall that $\tau$ was defined (in (3.1)) to be

$$
\tau=-\frac{d^{2}}{d r^{2}}+V(r) \quad(r>0) .
$$

The equation $\left(\tau-z^{2}\right) \phi(\cdot, z)=0$ may be written equivalently as

$$
w^{\prime}(r, z)=\left(\begin{array}{cc}
0 & 1 \\
V(r)-z^{2} & 0
\end{array}\right) w(r, z),
$$

where $w$ takes values in $\mathbf{C}^{2}(=\mathbf{C} \times \mathbf{C})$. Of course, $w$ and $\phi$ are related by the equation $w=\left(\begin{array}{c}\phi \\ \phi^{\prime}\end{array}\right)$. Therefore, we can study the solutions $w$ of $(4.2)$ and deduce the properties of $\phi$ ( $\phi$ will be the function in $\operatorname{Cond}(\xi)$ ).

The proof of Theorem 3.4 involves transforming iteratively the dependent variable in (4.2) with the object of reducing the coefficient matrix to a diagonal matrix plus a negligible error term. The asymptotic behavior of the transformed variable can then be determined by the method of Levinson [13]. The transformations are carried out in Lemma 4.1 below, but first we shall define what was referred to above as a "negligible error term".

Define $\mathcal{X}$ to be the set of all continuous functions, $x: U \rightarrow \mathbf{C}$ where $U$ is open in $\mathbf{R}_{+} \times \mathbf{C}_{j}$ (where $j \in \mathbf{N}$ depends on $x$, but $j=0$ if $\alpha \neq 1$ ) such that for every compact subset $K$ of $\mathbf{C}_{j}$ there is $r_{0}>0$ so that $\left(r_{0}, \infty\right) \times K \subseteq U$ and:

(1) for fixed $r>r_{0}$ the mapping $\lambda \mapsto x(r, \lambda)$ is continuously differentiable on $K \cap \mathbf{R}$;

(2) there are constants $C>0$ and $\varepsilon>0$ so that

$$
|x(r, z)|<C r^{-2-\varepsilon} \text { for all }(r, z) \in\left(r_{0}, \infty\right) \times K
$$

and

$$
\left|\frac{d}{d \lambda} x(r, \lambda)\right|<C r^{-2-\varepsilon} \text { for all }(r, \lambda) \in\left(r_{0}, \infty\right) \times\left(K \cap \mathbf{R}_{+}\right) .
$$

Observe that if $f \in E\left(\delta_{1}, \delta_{2}\right)$ where $\delta_{1}>2$ and $\delta_{2}>0$, then $f \in \mathcal{X}$. (See Lemma 3.2 and (3.2).) Also, if $f \in A$ and $x \in \mathcal{X}$, then $f x \in \mathcal{X}$.

Let $B\left(C^{2}\right)$ be the space of linear mappings on $C^{2}$. As usual $B\left(C^{2}\right)$ will be identified with the space of $2 \times 2$ matrices. Denote by $\chi^{4}$ the set of all $B\left(C^{2}\right)$ valued mappings, all of whose entries in the matrix representation belong to $\mathcal{X}$. Similarly, $A^{4}$ will denote the set of $B\left(\mathbf{C}^{2}\right)$-valued mappings with entries in $A$.

The transformations of $w$ in (4.2) are carried out in the following lemma.

LEMMA 4.1. Let $V, \delta_{1}$, and $\delta_{2}$ be as in assumption (V3). Let

$$
P(z)=\left(\begin{array}{cc}
1 & 1 \\
i z & -i z
\end{array}\right) \text {. }
$$

Then there exist $\xi \in E\left(\delta_{1}, \delta_{2}\right), S \in A^{4}$ and $T$ and $Y$ in $\mathfrak{X}^{4}$ so that if $v$ is a solution of

$$
v^{\prime}(r, z)=\left[i z(1+\xi(r, z))\left(\begin{array}{cc}
1 & 0 \\
0 & -1
\end{array}\right)+Y(r, z)\right] v(r, z)
$$


then $w$ defined by

$$
w(r, z)=P(z)(I+S(r, z)+T(r, z)) v(r, z)
$$

is a solution of (4.2). Moreover, $\xi=\bar{\xi}=\xi_{-}$.

Here $I$ denotes the identity in $B\left(\mathbf{C}^{2}\right)$. We shall leave aside the proof of Lemma 4.1 for the moment and show how it implies Theorem 3.4. A remark should be made at the outset.

REMARK 4.2. Suppose that, for some $\eta_{1}>0$ and $\eta_{2}>0$, $\xi \in E\left(\eta_{1}, \eta_{2}\right)$ (with domain $\mathbf{R}_{+} \times \mathbf{C}_{j}$ say $(j \in \mathbf{N})$ ) and that $\xi=\bar{\xi}$. This last condition implies that $\xi(r, \lambda)$ is real whenever $\lambda$ is real. Therefore, if $K$ is any compact subset of $\mathbf{C}_{j}$, there is $r_{0}>0$ and $\kappa>1$ so that, for all $(\sigma, z) \in\left(r_{0}, \infty\right) \times(K \backslash \mathbf{R})$

$$
\frac{1}{\kappa}<(\operatorname{Im} z)^{-1} \operatorname{Im}\{z+\xi(\sigma, z)\}<\kappa .
$$

This follows from Lemma 3.2, part (v) and (3.2).

Proof of Theorem 3.4. It suffices to construct a solution, $v$, of (4.3) such that

$$
v(r, z)=\exp \left\{i z\left(r+\int_{1}^{r} \xi(\sigma, z) d \sigma\right)\right\}\left(\begin{array}{l}
1 \\
0
\end{array}\right)+x(r, z)
$$

where both components of $x$ belong to $X$. Transforming back via (4.4) will give $\phi$ of $\operatorname{Cond}(\xi)$. In fact, it suffices to prove that such a solution $v$ exists on $\left(r_{0}, \infty\right) \times K$ for an arbitrary compact subset $K$ of $\mathbf{C}_{j}^{+}$and some $r_{0}>0$ because $\phi$ can be extended to $\mathbf{R}_{+} \times \mathbf{C}_{j}^{+}$by standard techniques (here $j \in \mathbf{N}$ is chosen so that $\xi, S, T$, and $Y$ of Lemma 4.1 are defined on $\mathbf{R}_{+} \times \mathbf{C}_{j}$ ). The required solution $v$ can be constructed by Levinson's method [13] or see [16, p. 2410] for a similar construction. This concludes the proof.

5. A related first order equation. To complete the proof of Theorem 3.4, it remains to prove Lemma 4.1; this requires some preparation. We begin with a technical lemma (Lemma 5.1) and then proceed to study the asymptotic behavior of solutions of a first order equation which arises in the iterative scheme used to prove Lemma 4.1.

LeMMA 5.1. Let $V_{L 2}, \delta_{1}>0$ and $\delta_{2}, 0<\delta_{2} \leqslant 1$, be as in Lemma 2.1. For each $m \in \mathbf{N}$, let $\mathcal{E}(m)$ denote the smallest subspace of $E\left(m \delta_{1}, \delta_{2}\right)$ containing functions of the form

$$
f(r, z)=f_{0}(r, z)\left(\int_{r}^{\infty} V_{L .2}(\sigma) d \sigma\right)^{k}
$$

for some $k \in \mathbf{N}$ and $f_{0} \in E_{0}\left((m-k) \delta_{1}, \delta_{2}\right)$. If $f \in \mathcal{E}(m)$ and $g \in \mathcal{E}(n)$,

$$
f g \in \mathcal{E}(m+n) \quad(m, n \in \mathbf{N}) .
$$

Moreover, if $f \in \mathcal{E}(m)$, then there is $\eta \in E\left((m+1) \delta_{1}+\delta_{2}, \delta_{2}\right)$ so that the integral $\int_{1}^{\infty} V_{L 2}(s) f(s, z)-\eta(s, z) d s$ exists conditionally and the function

$$
(r, z) \rightarrow \int_{r}^{\infty} V_{L 2}(s) f(s, z)-\eta(s, z) d s
$$

belongs to $\mathcal{E}(m+1)$. 
Proof. Because $\int_{r}^{\infty} V_{L 2}(s) d s$ belongs to $B\left(\delta_{1}, \delta_{2}\right)\left(\subseteq E\left(\delta_{1}, \delta_{2}\right)\right), \quad \mathcal{E}(m) \subseteq$ $E\left(m \delta_{1}, \delta_{2}\right)$ (see Lemma 3.2). Again by Lemma 3.2, $f \in \mathcal{E}(m)$ and $g \in \mathcal{E}(n)$ implies $f g \in \mathcal{E}(m+n)$. To verify the final statement it suffices to consider $f \in \mathcal{E}(m)$ of the form $f(r, z)=f_{0}(r, z)\left(\int_{r}^{\infty} V_{L 2}(\sigma) d \sigma\right)^{k}$ where $k \in \mathbf{N}$ and $f_{0} \in E_{0}\left((m-k) \delta_{1}, \delta_{2}\right)$. Integrate by parts

$$
\begin{aligned}
\int_{r}^{\infty} V_{L 2}(s) f(s, z) d s= & f_{0}(r, z)\left(\int_{r}^{\infty} V_{L 2}(\sigma) d \sigma\right)^{k+1} \\
& +\int_{r}^{\infty} f_{0}^{\prime}(s, z)\left(\int_{s}^{\infty} V_{L 2}(\sigma) d \sigma\right)^{k+1} d s .
\end{aligned}
$$

The result now follows by choosing $\eta(s, z)=f_{0}^{\prime}(s, z)\left(\int_{s}^{\infty} V_{L 2}(\sigma) d \sigma\right)^{k+1}$.

Consider the differential equation,

$$
u^{\prime}(r, z)=2 i z\left(1+\xi_{0}(r, z)\right) u(r, z)+\omega(r, z) \quad(r>0) .
$$

We shall show that if $\xi_{0}$ and $\omega$ belong to $E$ then there is a solution of (5.1) which belongs, except for a small error to $E$. By "a small error" we mean a function in $\mathscr{X}$ ( $\chi$ was defined prior to Lemma 4.1). More precisely, the result is as follows.

Lemma 5.2. Suppose that, for some $\eta_{1}>0, \delta_{1}>0$ and $\delta_{2}, 0<\delta_{2} \leqslant 1, \xi_{0} \in$ $E\left(\eta_{1}, \delta_{2}\right), \xi_{0}=\bar{\xi}_{0}$, and $\omega=\omega_{1}+\omega_{2}$ where

(1) $\omega_{1} \in E\left(\delta_{1}, \delta_{2}\right)$

(2) if $\alpha \leqslant 1$, then $\omega_{2}=0$, but if $\alpha>1$, then $\int_{1}^{\infty} \omega_{2}(s, z) d s$ exists conditionally and the function $(r, z) \mapsto \int_{r}^{\infty} \omega_{2}(s, z) d s$ belongs to $E\left(\delta_{1}, \delta_{2}\right)$.

Then there is $q \in E\left(\delta_{1}, \delta_{2}\right)$, with domain $\mathbf{R}_{+} \times \mathbf{C}_{j}$ say (i.e. define $\left.j \in \mathbf{N}\right)$ and $u$ : $\mathbf{R}_{+} \times \mathbf{C}_{j} \rightarrow \mathbf{C}$ which is a solution of (5.1) such that $u=q+\varepsilon$ where $\varepsilon: \mathbf{R}_{+} \times \mathbf{C}_{j} \rightarrow \mathbf{C}$ is continuous and the function $(r, z) \rightarrow r^{\alpha} \varepsilon(r, z)$ belongs to $\mathcal{X}$. More can be said if $\alpha>1$ : $q(r, z)=-\int_{r}^{\infty} \omega_{2}(s, z) d s+p_{0}(r, z)+p_{1}(r, z)$ where $p_{0} \in E_{0}\left(\delta_{1}, \delta_{2}\right)$ and $p \in$ $E\left(\delta_{1}+(\alpha-1), \delta_{2}\right)$.

Before giving the proof of Lemma 5.2 we shall show how it can be extended. Recall ((3.3a) and (3.3b)) the notations $\bar{f}$ and $f_{-}(f \in E)$.

COROllary 5.3. Assume the hypotheses and notation of Lemma 5.2. If in addition, $\xi_{0}=\bar{\xi}_{0}=\xi_{0-}$ and $\bar{\omega}=\omega_{-}$, then $q$ can be chosen so that $\bar{q}=q_{-}$.

Proof of Corollary 5.3. If $\bar{u}_{-}(r, z)=\overline{u(r,-\bar{z})}$ where $u$ is a solution of (5.1), then $\bar{u}_{-}$is also a solution of (5.1). Hence, replacing $u, q$, and $\varepsilon$ of Lemma 5.2 by $\frac{1}{2}\left(u+\bar{u}_{-}\right), \frac{1}{2}\left(q+\bar{q}_{-}\right)$and $\frac{1}{2}\left(\varepsilon+\bar{\varepsilon}_{-}\right)$(which does not affect the conclusion of that result) this corollary follows.

We turn now to the proof of Lemma 5.2. Several remarks should be made at the outset.

REMARK 5.4. (a) It is enough to prove Lemma 5.2 in the case $\omega \in E\left(\delta_{1}, \delta_{2}\right)$ (i.e., $\omega_{2}=0$ ). To show this, observe that $u$ is a solution of (5.1) if and only if $v$, defined by $v(r, z)=u(r, z)+\int_{r}^{\infty} \omega_{2}(s, z) d s$ is a solution of

$v^{\prime}(r, z)=2 i z\left(1+\xi_{0}(r, z)\right) v(r, z)+\omega_{1}(r, z)-2 i z\left(1+\xi_{0}(r, z)\right) \int_{r}^{\infty} \omega_{2}(s, z) d s$. 
The function $\omega_{1}(r, z)-2 i z\left(1+\xi_{0}(r, z)\right) \int_{r}^{\infty} \omega_{2}(s, z) d s$ belongs to $E\left(\delta_{1}, \delta_{2}\right)$ (by Lemma 3.2) so that the special case of Lemma 5.2 (when $\omega \in E\left(\delta_{1}, \delta_{2}\right)$ ) applies to give a particular solution $v$ of the above equation. Setting $u(r, z)=v(r, z)-$ $\int_{r}^{\infty} \omega_{2}(s, z) d s$ gives the desired solution of $(5.1)$.

(b) Furthermore, it is enough to consider $\omega \in E\left(\delta_{1}, \delta_{2}\right)$ of the form

$$
\omega(r, z)=e^{i k \beta r^{\alpha}} \omega_{0}(r, z) \quad \text { where } \omega_{0} \in E_{0}\left(\delta_{1}, \delta_{2}\right), k \in \mathbf{Z} .
$$

This follows because in general $\omega$ is a finite sum of such functions and because the solutions of (4.1) depend linearly on the nonhomogeneous term $\omega$.

(c) There is no difficulty solving (5.1); its general solution is

$$
u(r, z)=e^{i \theta(r, z)}\left\{c(z)+\int_{1}^{r} e^{-i \theta(s, z)} \omega(s, z) d s\right\}
$$

where

$$
\theta(r, z)=2 z\left(r+\int_{1}^{r} \xi_{0}(s, z) d s\right)
$$

for some $c(z) \in \mathbf{C}$.

Proof of Lemma 5.2. The desired expression for $u$ is obtained by expanding the integral in (5.3a) (where $\omega$ is defined by (5.2)) by repeated integration by parts; the factor $\exp \left\{-i\left(z s-k \beta s^{\alpha}\right)\right\}$ being integrated. At each stage this introduces into the integrand the factor

$$
h(s, z)=i\left(z-k \beta \alpha s^{\alpha-1}\right)^{-1} .
$$

We would like $h \in E_{0}(\max (0, \alpha-1), 1)$ because then Lemma 3.2(vi) could be applied to calculate derivatives. Indeed, when $\alpha=1, h(s, z)=h(z)$ is holomorphic except when $z=\frac{1}{2} k \beta$; i.e., $h \in D\left(\subseteq E_{0}(0,1)\right)$. (Incidentally, it is here we see the need to give special consideration to the points $z=\frac{1}{2} k \beta$ in the definition of $D$ when $\alpha=1$.) However, when $\alpha \neq 1, h \notin E_{0}(\max (0, \alpha-1), 1)$, but expanding $h$ in powers of $s^{-|\alpha-1|}$ (geometric series) we see $h$ is arbitrarily close to such a function. After suitably many integrations we obtain

$$
u(r, z)=q(r, z)+e^{i \theta(r, z)}\left\{c_{0}(z)+\int_{1}^{r} e^{-i \theta(s, z)} \varepsilon_{0}(s, z) d s\right\}
$$

for some $c_{0}(z) \in \mathbf{C}, \varepsilon_{0} \in E\left(\delta, \delta_{2}\right)$ where $\delta$ is large ( $\delta>7+2 \alpha$ suffices) and $g \in E$ which satisfies the required properties. The desired solution $u$ is obtained by choosing $c_{0}(z)$ (as we are still free to do) to be

$$
c_{0}(z)= \begin{cases}-\sum_{m=0}^{n} \frac{1}{m !} \int_{1}^{\infty} e^{-i \theta(s, \bar{z})}(4 \mu s)^{m} \varepsilon_{0}(s, z) d s, & \text { if } \mu \geqslant 0, \\ -\int_{1}^{\infty} e^{-i \theta(s, z)} \varepsilon_{0}(s, z) d s, & \text { if } \mu<0,\end{cases}
$$

where $\mu=\operatorname{Im} z$ and $n$ is large $(n \geqslant \alpha+2)$. With this choice of $c$ it is not difficult to check that $\varepsilon=u-q$ satisfies the required properties (for a similar argument, see [11, p. 597]). This coneludes the proof. 
6. Proof of Lemma 4.1. We shall now establish Lemma 4.1 and thus complete the proof of Theorem 3.4. Afterwards we shall indicate how the function $\xi$ there may be estimated. The proof of Lemma 4.1 involves transforming iteratively the dependent variable $w$ in the equation

$$
w^{\prime}(r, z)=\left(\begin{array}{cc}
0 & 1 \\
V(r)-z^{2} & 0
\end{array}\right) w(r, z)
$$

by a method introduced by Harris and Lutz [25] and extended by Ben-Artzi and Devinatz [11]. Ben-Artzi [9] obtained a similar result (when $\alpha<1$ ) by transforming the independent variable $r$.

Proof of Lemma 4.1. Suppose $w$ is a solution of (4.2). Then $u$ defined by $w(r, z)=P(z) u_{0}(r, z)$ is a solution of

$$
u_{0}^{\prime}(r, z)=\left[i z\left(1-\frac{1}{2} z^{-2} V(r)\right)\left(\begin{array}{cc}
1 & 0 \\
0 & -1
\end{array}\right)-\frac{i}{2} z^{-1} V(r)\left(\begin{array}{cc}
0 & 1 \\
-1 & 0
\end{array}\right)\right] u_{0}(r, z) .
$$

Let $V_{L}=V_{L 1}+V_{L 2}$ be the decomposition of $V_{L}$ of Lemma 2.1. ( $V_{L}$ as in (V3).) Let $\zeta_{0}(r, z)=i(2 z)^{-1} \int_{r}^{\infty} V_{L 2}(s) d s$ so that $\zeta_{0} \in E\left(\delta_{1}, \delta_{2}\right)$ and $\bar{\zeta}_{0}=\left(\zeta_{0}\right)_{-}=-\zeta_{0}$ (notation (3.3a) and (3.3b)). Define $v_{0}$ by the equation

$$
u_{0}=\left(\begin{array}{cc}
e^{\zeta_{0}} & 0 \\
0 & e^{-\zeta_{0}}
\end{array}\right) v_{0}
$$

so that

$$
\begin{array}{r}
v_{0}^{\prime}(r, z)=\left[i z\left(1-\frac{1}{2} z^{-2}\left(V_{L 1}(r)+V_{s}(r)\right)\left(\begin{array}{cc}
1 & 0 \\
0 & -1
\end{array}\right)-\frac{i}{2 z} V(r)\right)\right. \\
\left.\cdot\left(\begin{array}{cc}
0 & e^{-2 \zeta_{0}(r, z)} \\
-e^{2 \zeta_{0}(r, z)} & 0
\end{array}\right)\right] v_{0}(r, z) .
\end{array}
$$

Observe that

$$
e^{\zeta_{0}(r, z)}=1+\sum_{k=1}^{n} \frac{1}{k !}\left(\zeta_{0}(r, z)\right)^{k}+\sum_{k=n+1}^{\infty} \frac{1}{k !}\left(\zeta_{0}(r, z)\right)^{k}
$$

and that the finite sum belongs to $E\left(\delta_{1}, \delta_{2}\right)(\subseteq A)$ and the infinite sum belongs to $\chi$ provided $n$ is chosen large enough. Hence the transformation in (6.1) is of the form $I+S+T$ where $S \in A^{4}$ and $T \in \mathcal{X}^{4}$. Similarly, expanding $e^{-2 \zeta_{0}(r, z)}$ and $e^{2 \zeta_{0}(r, z)}$ into their Maclaurin series we see that the above equation for $v_{0}^{\prime}$ may be rewritten as

$$
v_{0}^{\prime}(r, z)=\left[i z\left(1+\xi_{0}(r, z)\right)\left(\begin{array}{cc}
1 & 0 \\
0 & -1
\end{array}\right)+\left(\begin{array}{cc}
0 & \omega_{0}(r, z) \\
\bar{\omega}_{0}(r, z) & 0
\end{array}\right)+Y_{0}\right] v_{0}(r, z)
$$

where $\xi_{0}(r, z)=-\frac{1}{2} z^{-2} V_{L 1}(r) ; \quad \omega_{0}(r, z)=-\frac{1}{2} i z^{-1} V_{L}(r) \sum_{k=0}^{l} \frac{1}{k !}\left(-2 \zeta_{0}(r, z)\right)^{k} \quad($ for some $l \in \mathbf{N})$; and $Y_{0} \in \mathcal{X}^{4}$. $\left(V_{S}\right.$ has been incorporated in $Y_{0}$.)

We shall transform $v_{0}$ in (6.2) iteratively. After $m-1$ transformations $(m \geqslant 1)$ the new variable, $v_{m-1}$ will be a solution of

$$
v_{m-1}^{\prime}(r, z)=\left[\Lambda_{m-1}(r, z)+W_{m-1}(r, z)+Y_{m-1}(r, z)\right] v_{m-1}(r, z)
$$


where

$$
\Lambda_{m-1}(r, z)=i z\left(1+\xi_{m-1}(r, z)\right)\left(\begin{array}{cc}
1 & 0 \\
0 & -1
\end{array}\right)
$$

and

$$
W_{m-1}(r, z)=\left(\begin{array}{cc}
0 & \omega_{m-1}(r, z) \\
\bar{\omega}_{m-1}(r, z) & 0
\end{array}\right)
$$

and $Y_{m-1} \in \mathcal{X}^{4}$. The functions $\xi_{m-1}$ and $\omega_{m-1}$ will satisfy:

(a) $\xi_{m-1}=\bar{\xi}_{m-1}=\left(\xi_{m-1}\right)_{-} ; \bar{\omega}_{m-1}=\left(\omega_{m-1}\right)_{-}$;

(b) $\xi_{m-1} \in E\left(\delta_{1}, \delta_{2}\right) ; \xi_{m-1}-\xi_{m-2} \in E\left(23^{m-2} \delta_{1}, \delta_{2}\right)$;

(c) $\omega_{m-1}(r, z)=V_{L 2}(r) \lambda_{m-1}(r, z)+\mu_{m-1}(r, z)$ where $\mu_{m-1} \in E\left(3^{m-1} \delta_{1}, \delta_{2}\right)$ and $\lambda_{m-1} \in \mathcal{E}\left(3^{m-1}-1\right) ;\left(\mathcal{E}\left(3^{m-1}-1\right)\right.$ is the subspace of $E\left(\left(3^{m-1}-1\right) \delta_{1}, \delta_{2}\right)$ defined in Lemma 5.1). In particular, $\omega_{m-1} \in E\left(3^{m-1} \delta_{1}, \delta_{2}\right)$ if $\alpha \leqslant 1$ (so that $V_{L 2}=0$ ) and $\omega_{m-1} \in E\left(3^{m-1} \delta_{1}-(\alpha-1), \delta_{2}\right)$ if $\alpha>1$.

Suppose that, in addition to obtaining $v_{m-1}$ with the above properties, we also show that, for $m \geqslant 2$

$$
v_{m-2}(r, z)=\left(I+S_{m-1}(r, z)+T_{m-1}(r, z)\right) v_{m-1}(r, z)
$$

for some $S_{m-1} \in A^{4}$ and $T_{m-1} \in \mathfrak{X}^{4}$. Then the result will follow. To verify this simply choose $m$ so large that $3^{m-1} \delta_{1}-\max (0, \alpha-1)>2$; this ensures that $W_{m-1}$ $\in \mathfrak{X}^{4}$. Defining $Y=W_{m-1}+Y_{m-1}$ and $\xi=\xi_{m-1}$ gives the result because the composition of transformations of the form " $I+S+T$ " is of the same form.

We shall now verify (6.3) by induction on $m$. When $m=1,(6.3)$ is just (6.2). It is clear that $\xi_{0}$ and $\omega_{0}$ satisfy (a) and (b) (take $\xi_{-1}=0$ in (b)). Moreover, $\omega_{0}$ satisfies (c): just choose

$$
\lambda_{0}(r, z)=-\frac{1}{2} i z^{-1} \sum_{k=0}^{l} \frac{1}{k !}\left(-2 \zeta_{0}(r, z)\right)^{k}
$$

(in $\left.\mathcal{E}(0) \subseteq E\left(0, \delta_{2}\right)\right)$ and $\mu_{0}(r, z)=V_{L 1}(r) \lambda_{0}(r, z)$ (in $\left.E\left(\delta_{1}, \delta_{2}\right)\right)$.

Suppose that the first $m-1$ transformations have been constructed with the required properties. We begin the construction of the $m$ th transformation by establishing the existence of a matrix $Q$ of the form

$$
Q(r, z)=\left(\begin{array}{cc}
0 & q_{12}(r, z) \\
q_{21}(r, z) & 0
\end{array}\right)
$$

which is a solution of the differential equation

$$
Q^{\prime}=\Lambda_{m-1} Q-Q \Lambda_{m-1}+W_{m-1}
$$

( $Q$ depends on $m$ although this is not indicated in the notation). Then $u_{m}$ will be defined by $v_{m-1}=R_{m} u_{m}$ where $R_{m}=\left(1-q_{12} q_{21}\right)^{1 / 2}(I+Q)$. Substituting into (6.3) we shall see that (6.4) was specified to reduce the off diagonal terms of the coefficient matrix as much as possible. A transformation analogous to (6.3) will take $u_{m}$ to $v_{m}$ which satisfies (6.3) with $m-1$ there replaced by $m$. 
Consider, therefore (6.4). Componentwise this equation becomes

$$
\begin{aligned}
& q_{12}^{\prime}(r, z)=2 i z\left(1+\xi_{m-1}(r, z)\right) q_{12}(r, z)+\omega_{m-1}(r, z), \\
& q_{21}^{\prime}(r, z)=-2 i z\left(1+\xi_{m-1}(r, z)\right) q_{21}(r, z)+\bar{\omega}_{m-1}(r, z) .
\end{aligned}
$$

Now equation (6.5a) is of the same form as (5.1) so that, in view of properties (a), (b), and (c) of $\xi_{m-1}$ and $\omega_{m-1}$, Lemma 5.2 is applicable (see also Lemma 5.1 to verify the required properties of $\left.\omega_{m-1}\right)$. Specify $q_{12}$ to be the solution of (6.5a) guaranteed by Lemma 5.2. Then

$$
q_{12}=q_{m}+\varepsilon
$$

where $q_{m} \in E\left(3^{m-1} \delta_{1}, \delta_{2}\right)$ and $\varepsilon$ is small in the sense that $r^{\alpha} \varepsilon(r, z)$ belongs to $\mathcal{X}$. Define $q_{21}=\bar{q}_{12}$. Then $q_{21}$ is a solution of $(6.5 \mathrm{~b})$ and

$$
q_{21}=\bar{q}_{m}+\bar{\varepsilon}
$$

Of course, $\bar{q}_{m} \in E\left(3^{m-1} \delta_{1}, \delta_{2}\right)$ by Lemma 3.2 and $r^{\alpha} \bar{\varepsilon}(r, z)$ belongs to $\mathcal{X}$. Moreover, by Corollary 5.3 and (a) it is possible to suppose that $\bar{q}_{m}=\left(q_{m}\right)_{-}$.

Define $R_{m}=\left(1-q_{12} q_{21}\right)^{-1 / 2}(I+Q)$ and $u_{m}$ by $v_{m-1}=R_{m} u_{m}$. Substituting into (6.3) and using the identity $(I+Q)^{-1}=I-(I+Q)^{-1} Q$ gives

$$
\begin{gathered}
u_{m}^{\prime}=\left[(I+Q)^{-1}\left\{\Lambda_{m-1}+\Lambda_{m-1} Q+\left(W_{m-1}+Y_{m-1}\right)(I+Q)-Q^{\prime}\right\}\right. \\
\left.-\frac{1}{2}\left(1-q_{12} q_{21}\right)^{-1}\left(q_{12}^{\prime} q_{21}+q_{12} q_{21}^{\prime}\right) I\right] u_{m} \\
=\left[\Lambda_{m-1}-\frac{1}{2}\left(1-q_{12} q_{21}\right)^{-1}\left(q_{12}^{\prime} q_{21}+q_{12} q_{21}^{\prime}\right) I\right. \\
+(I+Q)^{-1}\left\{\Lambda_{m-1} Q-Q \Lambda_{m-1}+W_{m-1}-Q^{\prime}\right. \\
\left.\left.+W_{m-1} Q+Y_{m-1}(I+Q)\right\}\right] u_{m} .
\end{gathered}
$$

Now $q_{12}^{\prime} q_{21}+q_{12} q_{21}^{\prime}=\omega_{m-1} q_{21}+q_{12} \bar{\omega}_{m-1}$ by (6.5a) and (6.5b). From this and (6.4) it follows that

$$
u_{m}^{\prime}=\left[M_{m}+X_{m}+Z_{m}\right] u_{m}
$$

where

$$
\begin{gathered}
M_{m}=\Lambda_{m}+\frac{1}{2}\left(1-q_{12} q_{21}\right)^{-1}\left(\omega_{m-1} q_{21}-\bar{\omega}_{m-1} q_{12}\right)\left(\begin{array}{cc}
1 & 0 \\
0 & -1
\end{array}\right), \\
X_{m}=-\left(1-q_{12} q_{21}\right)^{-1}\left(\begin{array}{cc}
0 & \bar{\omega}_{m-1}\left(q_{12}\right)^{2} \\
\omega_{m-1}\left(q_{21}\right)^{2} & 0
\end{array}\right)
\end{gathered}
$$

and $Z_{m}=(I+Q)^{-1} Y_{m-1}(I+Q)$.

Examine now the coefficient matrices, $M_{m}, X_{m}$, and $Z_{m}$. From (6.6a) and (6.6b) we see that, for some $\rho$

$$
\left(1-q_{12} q_{21}\right)^{-1}=\sum_{k=0}^{n}\left(q_{m} \bar{q}_{m}\right)^{k}+\rho
$$


and that $r^{\alpha} \rho(r, z)$ belongs to $\mathcal{X}$, provided $n \in \mathbf{N}$ is chosen large enough. (Fix $n$.) This property of $\rho$ assures that both $\rho$ and $r^{\alpha-1} \rho$ belong to $\mathcal{X}$ so that $\rho \in \mathcal{X}$ and $\rho \omega_{m-1} \in \mathcal{X}$. (Note if $\alpha>1$, then $\omega_{m-1}$ may be unbounded but $r^{-(\alpha-1)} \omega_{m-1}(r, z)$ is bounded locally uniformly in $z$.) Similarly, $\varepsilon_{m} \in \mathcal{X}$ and $\varepsilon_{m} \bar{\omega}_{m-1} \in \mathcal{X}$. Hence

$$
M_{m}=\Lambda_{m-1}+\frac{1}{2} \sum_{k=0}^{n}\left(q_{m} \bar{q}_{m}\right)^{k}\left(\omega_{m-1} \bar{q}_{m}-\bar{\omega}_{m-1} q_{m}\right)\left(\begin{array}{cc}
1 & 0 \\
0 & -1
\end{array}\right)+M_{m}^{0}
$$

and

$$
X_{m}=-\sum_{k=0}^{n}\left(q_{m} \bar{q}_{m}\right)^{k}\left(\begin{array}{cc}
0 & \bar{\omega}_{m-1}\left(q_{m}\right)^{2} \\
\omega_{m-1}\left(\bar{q}_{m}\right)^{2} & 0
\end{array}\right)+X_{m}^{0}
$$

where $X_{m}^{0} \in \mathcal{X}^{4}$ and $Y_{m}^{0} \in \mathcal{X}^{4}$. It is also clear that $Z_{m} \in \mathcal{X}^{4}$. From the expansion analogous to (6.8) for $\left(1-q_{12} q_{21}\right)^{-1 / 2}$ it follows that $R_{m}=\left(1-q_{12} q_{21}\right)^{-1 / 2}(I+Q)$ is of the form $I+S+T$ where $S \in A^{4}$ and $T \in \mathcal{X}^{4}$.

We shall isolate and then remove an unwanted part of $M_{m}$. By (c) $\omega_{m-1}=$ $V_{L 2} \lambda_{m-1}+\mu_{m-1}$. Because, by (a) $\bar{\omega}_{m-1}=\left(\omega_{m-1}\right)_{-}$, it is possible to suppose that $\bar{\lambda}_{m-1}=\left(\lambda_{m-1}\right)_{-}$and $\bar{\mu}_{m-1}=\left(\mu_{m-1}\right)_{-}$, for, if not, replace $\lambda_{m-1}$ and $\mu_{m-1}$ by $\frac{1}{2}\left(\lambda_{m-1}+\left(\bar{\lambda}_{m-1}\right)_{-}\right)$and $\frac{1}{2}\left(\mu_{m-1}+\left(\bar{\mu}_{m-1}\right)_{-}\right)$. According to Lemma 5.2, if $\alpha>1$, then

$$
q_{m}=p_{0}+p_{1}+p_{2}
$$

where $p_{0} \in E_{0}\left(3^{m-1} \delta_{1}, \delta_{2}\right), \quad p_{1} \in E\left(3^{m-1} \delta_{1}+\alpha-1, \delta_{2}\right)$ and $p_{2}(r, z)=$ $\int_{r}^{\infty} V_{L 2}(s) \lambda_{m-1}(s, z) d s$. Again it is possible to suppose that $\bar{p}_{0}=\left(p_{0}\right)_{-}$and $\bar{p}_{1}=$ $\left(p_{1}\right)_{-}$because $\bar{q}_{m}=\left(q_{m}\right)_{-}$. Define

$$
\theta_{m}=\frac{1}{2} \sum_{k=0}^{n}\left(\left(p_{0}+p_{2}\right)\left(\bar{p}_{0}+\bar{p}_{2}\right)\right)^{k}\left(\lambda_{m-1}\left(\bar{p}_{0}+\bar{p}_{2}\right)-\bar{\lambda}_{m-1}\left(p_{0}+p_{2}\right)\right)
$$

if $\alpha>1$ and $\theta_{m}=0$ otherwise; $V_{L 2} \theta_{m}$ is the unwanted part of $M_{m}$. Clearly, $\bar{\theta}_{m}=\left(\theta_{m}\right)_{-}=-\theta_{m}$.

By Lemma 5.1, $\theta_{m} \in \mathcal{E}\left(2 \cdot 3^{m-1}-1\right)$ and, for an appropriate choice of

$$
\eta \in E\left(2 \cdot 3^{m-1} \delta_{1}+\delta_{2}, \delta_{2}\right)
$$

$\zeta_{m}$ defined by

$$
\zeta_{m}(r, z)=-\int_{r}^{\infty} V_{L 2}(s) \theta_{m}(s, z)-\eta(s, z) d s
$$

belongs to $\mathcal{E}\left(2 \cdot 3^{m-1}\right)$. (If $\alpha \leqslant 1$, define $\zeta_{m}=0$.) It is possible to suppose that $\bar{\eta}=\eta_{-}=-\eta$, so that

$$
\bar{\zeta}_{m}=\left(\zeta_{m}\right)_{-}=-\zeta_{m},
$$

because if not replace $\eta$ by $\frac{1}{4}\left(\eta+\bar{\eta}_{-}-\bar{\eta}-\eta_{-}\right)$. Complete the $m$ th transformation by defining $v_{m}$ by

$$
u_{m}=\left(\begin{array}{cc}
e^{\zeta_{m}} & 0 \\
0 & e^{-\zeta_{m}}
\end{array}\right) v_{m}
$$


Now the argument following (6.1) can be repeated. We find that the above transformation is of the correct form (i.e., " $I+S+T$ "). Moreover, substituting into (6.7) gives

$$
\begin{aligned}
v_{m}^{\prime}(r, z)=\left[i z\left(1+\xi_{m}(r, z)\right)\right. & \left(\begin{array}{cc}
1 & 0 \\
0 & -1
\end{array}\right) \\
& \left.+\left(\begin{array}{cc}
0 & \omega_{m}(r, z) \\
\bar{\omega}_{m}(r, z) & 0
\end{array}\right)+Y_{m}(r, z)\right] v_{m}(r, z)
\end{aligned}
$$

where

$$
\begin{gathered}
\xi_{m}=\xi_{m-1}-i z^{-1}\left\{\frac{1}{2} \sum_{k=0}^{n}\left(q_{m} \bar{q}_{m}\right)^{k}\left(\omega_{m-1} \bar{q}_{m}-\bar{\omega}_{m-1} q_{m}\right)-V_{L 2} \theta_{m}+\eta\right\}, \\
\omega_{m}=-\bar{\omega}_{m-1}\left(q_{m}\right)^{2}\left(\sum_{k=0}^{n}\left(q_{m} \bar{q}_{m}\right)^{k}\right)\left(\sum_{j=0}^{l} \frac{1}{j !}\left(-2 \zeta_{m}\right)^{j}\right)
\end{gathered}
$$

(for some $l \in \mathbf{N}$ ) and $Y_{m} \in \mathcal{X}^{4}$. We have used (6.9) and (6.10) and incorporated $M_{m}^{0}$ and $X_{m}^{0}$ with $Z_{m}$.

It remains only to check that $\xi_{m}$ and $\omega_{m}$ satisfy (a), (b), and (c) with $m-1$ there replaced by $m$. Property (a) is immediate. For (b) it suffices to check that $\xi_{m}-\xi_{m-1}$ $\in E\left(2 \cdot 3^{m-1} \delta_{1}, \delta_{2}\right)$. Certainly $\eta \in E\left(2 \cdot 3^{m-1} \delta_{1}, \delta_{2}\right)$. Recall that

$$
\omega_{m-1} \in E\left(3^{m-1} \delta_{1}-(\alpha-1), \delta_{2}\right) \text { and } q_{m}=p_{0}+p_{1}+p_{2}
$$

where $p_{1} \in E\left(3^{m-1} \delta_{1}+\alpha-1, \delta_{2}\right)$ and $\left(p_{0}+p_{2}\right) \in E\left(3^{m-1} \delta_{1}, \delta_{2}\right)$. Expanding $\frac{1}{2} \sum_{k=0}^{n}\left(q_{m} \bar{q}_{m}\right)\left(\omega_{m-1} \bar{q}_{m}-\bar{\omega}_{m-1} q_{m}\right)$ in powers of $p_{1}$ and $\left(p_{0}+p_{2}\right)$ we see that every term involving at least one $p_{1}$ is in $E\left(2 \cdot 3^{m-1} \delta_{1}, \delta_{2}\right)$. The remaining term is

$$
V_{L 2} \theta_{m}+\frac{1}{2} \sum_{k=0}^{n}\left(\left(p_{0}+p_{2}\right)\left(\bar{p}_{0}+\bar{p}_{2}\right)\right)^{k}\left(\mu_{m-1} \bar{q}_{m}-\bar{\mu}_{m-1} q_{m}\right)
$$

because $\omega_{m-1}=V_{L 2} \lambda_{m-1}+\mu_{m-1}$. The $V_{L 2} \theta_{m}$ cancels in (6.11) and the sum belongs to $E\left(2 \cdot 3^{m-1} \delta_{1}, \delta_{2}\right)$; this proves (b). The proof of (c) is similar. It suffices to say that $\lambda_{m}$ should be defined as

$$
\lambda_{m}=-\bar{\lambda}_{m-1}\left(p_{0}+p_{2}\right)^{2}\left(\sum_{k=0}^{n}\left(\left(p_{0}+p_{2}\right)\left(\bar{p}_{0}+\bar{p}_{2}\right)\right)^{k}\right)\left(\sum_{j=0}^{l} \frac{1}{j !}\left(-2 \zeta_{m}\right)^{j}\right) .
$$

This completes the proof of Lemma 4.1.

There are several further consequences of the above proof that are worthwhile recording.

Lemma 6.1. Assume the hypotheses and notation of Theorem 3.4, and let $V_{L 1}$ and $V_{L 2}$ be as in Lemma 2.1. Then for some $x \in E\left(2 \delta_{1}, \delta_{2}\right)$

$$
\xi(r, z)=-\left(2 z^{2}\right)^{-1} V_{L 1}(r)+x(r, z) .
$$


Proof. By Theorem 3.4, $V_{L}$ satisfies Cond $(\xi)$ for some $\xi \in E$. In the notation of the previous proof, $\xi=\xi_{m}$ for some $m \in \mathbf{N}$. Write $\xi_{m}=\xi_{0}+x$ where $x=\sum_{k=1}^{m} \xi_{k}$ $-\xi_{k-1}$. Then $x \in E\left(2 \delta_{1}, \delta_{2}\right)$ by (b) of the previous proof and since $\xi_{0}(r, z)=$ $-\left(2 z^{2}\right)^{-1} V_{L 1}(r)$ the result follows.

Corollary 6.2. Assume the hypothesis of Lemma 6.1. If in addition $\int_{1}^{\infty} V_{L 1}(s) d s$ exists conditionally and for some $\varepsilon>0$,

$$
\int_{r}^{\infty} V_{L 1}(s) d s=O\left(r^{-\varepsilon}\right) \text { as } r \rightarrow \infty,
$$

then $V_{L}$ satisfies $\operatorname{Cond}(\xi)$ for some $\xi$ in $E\left(2 \delta_{1}, \delta_{2}\right)$.

Proof. This result follows from the previous by way of Lemma 3.3.

REMARK 6.3. It is possible to refine the estimate $\xi$ in Lemma 6.1 by estimating the function $\xi_{1}$ in the proof of Lemma 4.1. To state the estimate we need some notation. Recall that $V_{L 2} \in B\left(\delta_{1}, \delta_{2}\right)$ and $\int_{r}^{\infty} V_{L 2}(\sigma) d \sigma$ also belong to $B\left(\delta_{1}, \delta_{2}\right)$ and is oscillatory so that

$$
\begin{gathered}
V_{L 1}(r)=g_{0}^{1}(r)+\sum_{k=1}^{n} g_{k}^{1}(r) \cos k \beta r^{\alpha}+f_{k}^{1}(r) \sin k \beta r^{\alpha}, \\
\int_{r}^{\infty} V_{L 2}(s) d s=\sum_{k=1}^{n} g_{k}^{2}(r) \cos k \beta r^{\alpha}+f_{k}^{2}(r) \sin k \beta r^{\alpha}
\end{gathered}
$$

for some $n \in \mathbf{N}$ and some function $g_{k}^{1}$ and $f_{k}^{1}$ in $C\left(\delta_{1}, \delta_{2}\right)$. In terms of this notation there is $x \in E\left(\delta, \delta_{2}\right)$ where $\delta>2 \delta_{1}$ so that the function $\xi$ of Lemma 6.1 is:

(1) if $\alpha<1$, then

$$
\xi(r, z)=-\left(2 z^{2}\right)^{-1} V_{L 1}(r)\left\{1+(2 z)^{-2} V_{L 1}(r)\right\}+x(r, z) ;
$$

(2) if $\alpha=1$, then

$$
\begin{aligned}
\xi(r, z)= & -\left(2 z^{2}\right)^{-1} V_{L 1}\{1+ \\
& +(2 z)^{-2} g_{0}^{1}(r) \\
& \left.+\sum_{k=1}^{n}\left(4 z^{2}-k^{2} \beta^{2}\right)^{-1}\left(g_{k}^{1}(r) \cos k \beta r+f_{k}^{1}(r) \sin k \beta r\right)\right\} \\
& +x(r, z)
\end{aligned}
$$

(3) if $\alpha>1$, then

$$
\begin{aligned}
\xi(r, z)= & -\left(2 z^{2}\right)^{-1} V_{L 1}(r)\left\{1+(2 z)^{-2} g_{0}^{1}(r)\right\}+\left(\beta \alpha r^{\alpha-1}\right)^{-1} V_{L 2}(r) \\
& \cdot \sum_{k=1}^{n} \frac{1}{k}\left(f_{k}^{2}(r) \cos k \beta r^{\alpha}-g_{k}^{2}(r) \sin k \beta r^{\alpha}\right)+x(r, z) .
\end{aligned}
$$

From these estimates the behavior at $\infty$ of the generalized phase shift $\int_{1}^{r} \xi(\sigma, z) d \sigma$ (from (4) of Cond $(\xi)$ ) can be determined. This will be important when we consider existence of wave operators.

REMARK 6.4. Having established Lemma 4.1, we have verified Theorem 3.4 but only in case $\nu=0$. The general case follows by a minor alteration to the proof of 
Lemma 4.1. There is, however, a subtlety: We require that both $\xi$ and the domain of $\phi$ (in $\operatorname{Cond}(\xi))$ do not depend on $\nu$. We indicate therefore the necessary changes to the proof of Lemma 4.1.

In the general case $\nu \in \mathbf{R}$ the inductive hypothesis (replacing (6.3)) becomes

$$
v_{m-1}^{\prime}=\left[\Lambda_{m-1}+W_{m-1}+U+Y_{m-1}^{0}\right] v_{m-1}
$$

where

$$
U(r, z)=-i \nu(2 z)^{-1} r^{-2}\left(\begin{array}{cc}
1 & 1 \\
-1 & -1
\end{array}\right)
$$

and $\Lambda_{m-1}$ and $W_{m-1}$ are just as before and $Y_{m-1}^{0} \in \mathcal{X}^{4}$ may differ from $Y_{m-1}$. It is easy to check that the form of $(6.12)$ is preserved by the double transformations of Lemma 4.1 (which are exactly as before), and this verifies (6.12) for all $m \in \mathbf{N}$.

Applying (6.12) when $m$ is so large that $W_{m-1} \in \mathcal{X}^{4}$ gives

$$
\begin{aligned}
v_{m-1}^{\prime}(r, z)=\left[i z\left(1+\xi^{0}(r, z)\right)\right. & \left(\begin{array}{cc}
1 & 0 \\
0 & -1
\end{array}\right) \\
& \left.+\left(\begin{array}{cc}
0 & \omega(r, z) \\
\bar{\omega}(r, z) & 0
\end{array}\right)+Y^{0}\right] v_{m-1}(r, z)
\end{aligned}
$$

where $\xi^{0}(r, z)=\xi_{m-1}(r, z)-\left(2 z^{2}\right)^{-1} \nu r^{-2} ; \omega(r, z)=-i(2 z)^{-1} \nu r^{-2}$ and $Y^{0}=$ $W_{m-1}+Y_{m-1}^{0} \in X^{4}$. This equation is exactly like (6.3) with $\omega \in E(2,1)$. A transformation analogous to $R_{m}$ is applicable and leads to a new variable, $v$ say, satisfying an equation analogous to (6.7). This time the function $q_{m}$ which we denote $q^{0}$ belongs to $E\left(2, \delta_{2}\right)$. Hence, the analogue of $X_{m}$ belongs to $\chi^{4}$ (see (6.10)) as does the analogue of $M_{m}-\Lambda_{m-1}$. Hence,

$$
v^{\prime}(r, z)=\left[i z\left(1+\xi^{0}(r, z)\right)\left(\begin{array}{cc}
1 & 0 \\
0 & -1
\end{array}\right)+Y(r, z)\right] v(r, z)
$$

for some $Y \in \mathcal{X}^{4}$. Thus, Lemma 4.1 remains true when $V(r)$ is replaced by $\nu r^{-2}+V(r)$ and the proof of Theorem 3.4 applies to show $\nu r^{-2}+V(r)$ satisfies Cond $\left(\xi^{0}\right)$. By Lemma 3.3 it follows that $\nu r^{-2}+V(r)$ satisfies Cond $(\xi)$ (because $\left.\xi=\xi_{m-1}\right)$.

It remains to consider how the domain $D(\phi)$ of the function $\phi$ of $\operatorname{Cond}(\xi)$ depends on $\nu$. If $\alpha \neq 1$, then $D(\phi)=\mathbf{R}_{+} \times \mathbf{C}_{0}^{+}$which does not depend on $\nu$. If $\alpha=1$, then $D(\phi)=\mathbf{R}_{+} \times \mathbf{C}_{j}^{+}$where $j \in \mathbf{N}$ is so large that $\xi, Y, S$, and $T$ of Lemma 4.1 are defined on $\mathbf{R}_{+} \times \mathbf{C}_{j}$ (see the proof of Theorem 3.4). By the first part of this proof the only change in the domains of $\xi, Y, S$, and $T$ resulting from replacing $V(r)$ by $\nu r^{-2}+V(r)$ is caused by the last transformation. Hence $j$ need only be chosen so large that $q^{0}$ is also defined on $\mathbf{R}_{+} \times \mathbf{C}_{j}$. It is not difficult to check that the domain of $q^{0}$ does not depend on $\nu$. Thus Theorem 3.4 is completely established in the general case $\nu \in \mathbf{R}$.

One consequence of the above remark is that if $V=0\left(H=H_{0}\right)$ then $\xi$ in Theorem 3.4 may be chosen to be 0 . This follows because $\xi$ does not depend on $\nu$ and when $\nu=0, \operatorname{Cond}(\xi)$ is trivially satisfied with $\varphi(r, z)=\exp (i z r)$ and $\xi=0$. 
Thus we have

CorollaRy 6.5. For all $\nu \in \mathbf{R}$, the function $r \rightarrow \nu r^{-2}$ satisfies Cond(0).

7. Limiting absorption principle. Recall the operators $H(l)(l \in \mathrm{N})$ defined in $\S 2$ and the associated differential expressions $\tau_{l}$ of (2.1). We shall establish a limiting absorption principle for $H(l)$ and thereby derive the properties of its spectrum. We begin with a remark about the solutions of $\left(\tau_{l}-z^{2}\right) \phi=0$ when $z^{2}$ is real.

REMARK 7.1. (a) Suppose that $V$ satisfies $(V 1),(V 2)$, and (V3). Then, by Theorem $3.4, \nu(l) r^{-2}+V(r)$ satisfies $\operatorname{Cond}(\xi)(\xi \in E)$ for every $l \in \mathbf{N}$. Let $\phi_{l}: \mathbf{R}_{+} \times \mathbf{C}_{j}^{+} \rightarrow \mathbf{C}$ be the corresponding function of $\operatorname{Cond}(\xi)$ and observe that $j \in \mathbf{N}$ does not depend on $l$ (Theorem 3.4) and $j=0$ if $\alpha \neq 1$. Let $\lambda \in \mathbf{C}_{j} \cap \mathbf{R}_{+}$. Then $\xi(\cdot, \lambda)$ is real-valued, because $\xi=\bar{\xi}$ so that, by (4) of $\operatorname{Cond}(\xi), \phi_{l}(\cdot, \lambda)$ and $\overline{\phi_{l}(\cdot, \lambda)}$ are linearly independent solutions of $\left(\tau_{l}-\lambda^{2}\right) \phi=0$, and no nontrivial linear combination of them belongs to $L^{2}\left(\mathbf{R}_{+}\right)$.

(b) Let $\psi$ be the function of Lemma 3.1. If $\lambda \in \mathbf{C}_{j} \cap \mathbf{R}_{+}$, then $\psi(\cdot, \lambda)$ is another solution of $\left(\tau_{l}-\lambda^{2}\right) \phi=0$ and hence is a linear combination of $\phi_{l}(\cdot, \lambda)$ and $\overline{\phi_{l}(\cdot, \lambda)}$ but $\psi(\cdot, \lambda)$ is not a multiple of $\phi_{l}(\cdot, \lambda)$ (or of $\overline{\phi_{l}(\cdot, \lambda)}$ ) because $\psi(\cdot, \lambda)=\overline{\psi(\cdot, \lambda)}$.

(c) For every $\lambda>0$, there is a real solution of $\left(\tau_{l}+\lambda^{2}\right) \phi=0$ which has only finitely many zeros on $(1, \infty)$ : it is $\operatorname{Re} \phi_{l}(\cdot, i \lambda)$. This follows by (4) of $\operatorname{Cond}(\xi)$ because $\xi(\cdot, i \lambda)$ is real-valued (since $\xi=\bar{\xi}=\xi_{-}$). This and part (g) of Lemma 3.1 imply that all real solutions of $\left(\tau_{l}+\lambda^{2}\right) \phi=0$ have only finitely many zeros on $\mathbf{R}_{+}$. (See also [20, Lemma 35, p. 1462].)

Recall the notation $H^{\prime}, H=$ “ $-\Delta+V$ ”, $D\left(T_{1}\left(\tau_{l}\right)\right)$ and $B_{l}$ introduced in $\S 2$. We shall now show that $H$ is uniquely determined by $H^{\prime}$.

LEMma 7.2. If $V$ satisfies $(V 1),(V 2),\left(V 2^{\prime}\right)$ and $(V 3)$, then $H^{\prime}(=-\Delta+V$ on $\left.C_{0}^{\infty}\left(\mathbf{R}^{d}\right)\right)$ is essentially selfadjoint. Moreover, for each $l \in \mathbf{N}$, the operator $H(l)$ defined in $\S 2$ has domain $D(H(l))=\left\{f \in D\left(T_{1}\left(\tau_{l}\right)\right): B_{l} f=0\right\}$.

Proof. Establish the last conclusion first. Suppose, therefore, that $H$ is any selfadjoint extension of $H^{\prime}$ and let $H(l)$ be the corresponding operators defined in $\S 2$ so that $D(H(l)) \subseteq\left\{f \in D\left(T_{1}\left(\tau_{l}\right)\right): B_{l} f=0\right\}$. By Remark 7.1(a), not all solutions of $\left(\tau_{l}-\lambda^{2}\right) \phi=0$ belong to $L^{2}((1, \infty))$. It follows that $\tau_{l}$ has no boundary values at $\infty$. (See [20, Theorem 11, p. 1401]; take $I=[1, \infty)$ there.) On the other hand, the space of all solutions of $\left(\tau_{l}-z^{2}\right) \psi=0$, which belong to $L^{2}((0,1))$ and satisfy $B_{l} \psi=0$ is one dimensional by Lemma 3.1. Therefore $D(H(l))$ cannot be a proper subspace of $\left\{f \in D\left(T_{1}\left(\tau_{l}\right)\right): B_{l} f=0\right\}$ (see [20, Theorem 32, p. 1309]).

Therefore, $D(H(l))$ and hence $H(l)$ is uniquely determined no matter which selfadjoint extension $H$ of $H^{\prime}$ was chosen. Hence, $H^{\prime}$ can have only one selfadjoint extension which implies that $H^{\prime}$ is essentially selfadjoint. This completes the proof.

We leave aside consideration of $H=$ " $-\Delta+V$ " until $\S 9$ and proceed with the analysis of $H(l)$. We shall make use of the following general assumptions and notation.

Hypothesis 7.3. Suppose $V: \mathbf{R}_{+} \rightarrow \mathbf{R}$ satisfies $(V 1),(V 2),\left(V 2^{\prime}\right)$, and (V3). Fix $l \in \mathbf{N}$ and let $H(l)$ be the selfadjoint operator defined in $\$ 2$ and let $P$ be its spectral measure. 
Choose $\xi \in E$ so that the function $r \rightarrow \nu(l) r^{-2}+V(r)$ satisfies Cond( $\left.\xi\right)$ (Theorem 3.4) and let $\phi: \mathbf{R}_{+} \times \mathbf{C}_{j}^{+} \rightarrow \mathbf{C}$ be the function appearing in $\operatorname{Cond}(\xi)$. Let $\psi$ be the function from Lemma 3.1. Set $\mathbf{R}_{j}^{+}=\left\{\lambda>0: \sqrt{\lambda} \in \mathbf{C}_{j}\right\}=\mathbf{R}_{+}-\left\{\frac{1}{4}(k \beta)^{2}: 0 \leqslant k \leqslant j\right\}$.

Observe that, by Remark 7.1(a), if $\lambda \in \mathbf{R}_{j}^{+}$, then $\lambda$ is not an eigenvalue of $H(l)$. The next result is a limiting absorption principle for $H(l)$.

TheOREM 7.4. Assume Hypothesis 7.3. If the interval $\left(\lambda_{1}, \lambda_{2}\right)$ has closure in $\mathbf{R}_{j}^{+}$and if $f \in L^{2}\left(\mathbf{R}_{+}\right)$has compact support, then

$$
\left(P\left(\left(\lambda_{1}, \lambda_{2}\right)\right) f \mid f\right)=\frac{1}{\pi} \operatorname{Im}\left\{\int_{\lambda_{1}}^{\lambda_{2}} \int_{0}^{\infty} \int_{0}^{\infty} K(r, s ; \lambda) f(s) \overline{f(r)} d s d r d \lambda\right\}
$$

where

$$
K(r, s ; \lambda)= \begin{cases}a(\lambda) \psi(r, \sqrt{\lambda}) \phi(s, \sqrt{\lambda}) & \text { if } r \leqslant s \\ a(\lambda) \phi(r, \sqrt{\lambda}) \psi(s, \sqrt{\lambda}) & \text { if } r>s\end{cases}
$$

for some a: $\mathbf{R}_{j}^{+} \rightarrow \mathbf{C} \backslash\{0\}$ which is continuously differentiable. In fact, $a(\lambda)=$ $W(\phi(\cdot, \sqrt{\lambda}) \psi(\cdot, \sqrt{\lambda}))^{-1}$ where $W$ is the Wronskian.

Here $(\cdot \mid \cdot)$ denotes the inner product on $L^{2}\left(\mathbf{R}_{+}\right)$. Observe that the Wronskian, $W(\psi(\cdot, z), \psi(\cdot, z))=\phi(r, z) \psi^{\prime}(r, z)-\phi^{\prime}(r, z) \psi(r, z)$ does not depend on $r$ because $\phi(\cdot, z)$ and $\psi(\cdot, z)$ are solutions of $\left(\tau_{l}-z^{2}\right) \phi=0$. The argument below is due to Ben-Artzi [10].

Proof of Theorem 7.4. Two general results will be called upon. The first is Stieltjes' inversion formula [20, Theorem 11, p. 1203]. Because $\lambda_{1}$ and $\lambda_{2}\left(\right.$ in $\mathbf{R}_{j}^{+}$) are not eigenvalues of $H(l)$ (by Remark 1.7(a)) this formula may be written as

$$
\left(P\left(\lambda_{1}, \lambda_{2}\right) f \mid f\right)=\frac{1}{\pi} \lim _{\varepsilon \rightarrow 0+} \int_{\lambda_{1}}^{\lambda_{2}} \operatorname{Im}\left((H(l)-(\lambda+i \varepsilon) I)^{-1} f \mid f\right) d \lambda .
$$

The second result is that the resolvent operator is an integral operator

$$
(H-z I)^{-1} f(r)=\int_{0}^{\infty} K(r, s, z) f(s) d s
$$

with kernel

$$
K(r, s, z)= \begin{cases}a(z) \psi(r, \sqrt{z}) \phi(s, \sqrt{z}) & \text { if } r \leqslant s \\ a(z) \phi(r, \sqrt{z}) \psi(s, \sqrt{z}) & \text { if } r>s\end{cases}
$$

where $a(z)=W(\phi(\cdot, \sqrt{z}), \psi(s, \sqrt{z}))^{-1}$ and $\operatorname{Im} z>0$. (See [20, Theorem 16, p. 1329].)

Substituting into the equation for $\left(P\left(\lambda_{1}, \lambda_{2}\right) f \mid f\right)$ will give equation (6.1) provided we can justify taking the limit over $\varepsilon$ inside the triple integral. Since $\phi, \phi^{\prime}, \psi$ and $\psi^{\prime}$ are all continuous the only possible difficulty is that $W(\phi(\cdot, \sqrt{\lambda}), \psi(\cdot, \sqrt{\lambda}))$ may be 0 for some $\lambda \in\left(\lambda_{1}, \lambda_{2}\right)$. But $\psi(\cdot, \sqrt{\lambda})$ is not a multiple of $\phi(\cdot, \sqrt{\lambda})$ by Remark 7.1(b), so that this cannot happen. This justifies the expression (7.1) and the statement about $a$ is obvious: the proof is complete. 
Many properties of the spectrum of $H(l)$ can be inferred from Theorem 7.4. Recall, therefore, the relevant definitions. Let $H$ be a selfadjoint operator on a Hilbert space, $\mathcal{H}$. The essential spectrum $\sigma_{e}(H)$ is the spectrum of $H$ with all the isolated eigenvalues of finite multiplicity removed. If $P$ is the spectral measure of $H$ and $\Sigma$ is the Borel subsets of $\mathbf{R}$, then for each $f \in \mathcal{H}$,

$$
\mu_{f}(A)=(P(A) f \mid f) \text { for all } A \in \Sigma
$$

defines a measure on $\Sigma$. The subspace of absolute continuity $\mathcal{H}_{\mathrm{ac}}$ of $H$ is the set of all $f \in \mathcal{H}$ such that $\mu_{f}$ is absolutely continuous with respect to Lebesgue measure $m$ on $\Sigma$ (in symbols $\mu_{f} \ll m$ ); $\mathcal{H}_{\mathrm{ac}}$ is a closed subspace of $\mathcal{H}$ which reduces $H$. The restriction, $H_{\mathrm{ac}}$ of $H$ to $\mathcal{H}_{\mathrm{ac}}$ is the absolutely continuous part of $H$ and the spectrum $\sigma_{\mathrm{ac}}(H)$ is the absolutely continuous spectrum of $H$. Consequently, $\sigma_{\mathrm{ac}}(H) \subseteq \sigma_{e}(H)$. If the orthogonal complement of the space spanned by the eigenvalues of $H$ is $\mathcal{H}_{\mathrm{ac}}$, then $H$ has void singularly continuous spectrum. For a complete treatment of these concepts, see Chapter X, $\$ 1.2$ of Kato's book [31].

If two measures $\mu_{1}$ and $\mu_{2}$ on $\Sigma$ are absolutely continuous with respect to each other, i.e., $\mu_{1} \ll \mu_{2}$ and $\mu_{2} \ll \mu_{1}$, then we shall write $\mu_{1} \sim \mu_{2}$. Apparently, " $\sim$ " is an equivalence relation. We shall now derive two corollaries to Theorem 7.4.

Corollary 7.5. Assume Hypothesis 7.3 and denote by $L^{2}\left(\mathbf{R}_{+}\right)_{\mathrm{ac}}$ the subspace of absolute continuity of $H(l)$. If $\left(\lambda_{1}, \lambda_{2}\right) \subseteq \mathbf{R}_{j}^{+}$and $\lambda_{2}>\lambda_{1}$, then $P\left(\lambda_{1}, \lambda_{2}\right) L^{2}\left(\mathbf{R}_{+}\right)$is a nonzero subspace of $L^{2}\left(\mathbf{R}_{+}\right)_{\mathrm{ac}}$. Moreover, there is $f_{0} \in L^{2}\left(\mathbf{R}_{+}\right)_{\mathrm{ac}}$ so that $\mu_{f_{0}} \sim m$ where $m$ is Lebesgue measure on $\mathbf{R}_{+}$and 0 on $(-\infty, 0]$.

Proof. If $f \in L^{2}\left(\mathbf{R}_{+}\right)$has compact support and $g=P\left(\left(\lambda_{1}, \lambda_{2}\right)\right) f$, then $g \in$ $L^{2}\left(\mathbf{R}_{+}\right)_{\text {ac }}$ by (7.1). Hence, $P\left(\left(\lambda_{1}, \lambda_{2}\right)\right) L^{2}\left(\mathbf{R}_{+}\right) \subseteq L^{2}\left(\mathbf{R}_{+}\right)_{\mathrm{ac}}$. Moreover, by (7.1) the Radon-Nikodym derivative, $\rho_{g}$ of $\mu_{g}$ with respect to $m$ is

$$
\rho_{g}(\lambda)=\frac{1}{\pi} \chi_{\left(\lambda_{1}, \lambda_{2}\right)}(\lambda) \operatorname{Im}\left\{\int_{0}^{\infty} \int_{0}^{\infty} K(r, s ; \lambda) f(s) \overline{f(r)} d s d r\right\}
$$

$\left(\chi_{\left(\lambda_{1}, \lambda_{2}\right)}\right.$ denotes the indicator function for $\left.\left(\lambda_{1}, \lambda_{2}\right)\right)$. We shall now show that for arbitrary $\lambda_{0}$ there is $f$ (of compact support) so that $\rho_{g}\left(\lambda_{0}\right) \neq 0$. Since $K$ is continuous it suffices to show that there is a nondegenerate compact interval $I$ so that $\operatorname{Im} K\left(r, s ; \lambda_{0}\right) \neq 0$ for all $(r, s)$ in $I \times I$ : for simply choose $f=\chi_{I}$. To show this recall that $\psi\left(\cdot, \sqrt{\lambda_{0}}\right)$ is real-valued and has only discrete zeros (as a solution of $\left.\left(\tau_{l}-\lambda_{0}\right) \psi=0\right)$. Also, $\operatorname{Im}\left(a\left(\lambda_{0}\right) \phi\left(\cdot, \sqrt{\lambda_{0}}\right)\right)$ has discrete zeros by (4) of $\operatorname{Cond}(\xi)$. It is clear, therefore, how to choose $I$; hence $\rho\left(\lambda_{0}\right) \neq 0$. Since $\rho$ is continuous $g \neq 0$ and, therefore, $P\left(\lambda_{1}, \lambda_{2}\right) L^{2}\left(\mathbf{R}_{+}\right) \neq\{0\}$.

More can be concluded. Because $\lambda_{1}, \lambda_{2}$, and $\lambda_{0}$ were arbitrary it is possible to cover $\mathbf{R}_{j}^{+}$by a sequence $\left\{\Lambda_{n}\right\}_{n \in \mathbf{N}}$ of disjoint intervals such that for each $n$ there is $g_{n} \in L^{2}\left(\mathbf{R}_{+}\right)_{\text {ac }}$ so that the Radon-Nikodym derivative $\rho_{n}$ of $\mu_{g_{n}}$ is continuous and nonzero on $\Lambda_{n}$. Define $f_{0}=\Sigma_{n \in \mathrm{N}} 2^{-n} P\left(\Lambda_{n}\right) g_{n}$. Then $f_{0} \in L^{2}\left(\mathbf{R}_{+}\right)_{\text {ac }}$ and $\mu_{f_{0}} \sim m$. The proof is complete.

The properties of the spectrum of $H(l)$ may now be summarized. 
Corollary 7.6. Assume Hypothesis 7.3. Then $H(l)$ has no positive eigenvalues if $\alpha \neq 1$, but if $\alpha=1$, then $\left\{\frac{1}{4}(k \beta)^{2}: 1 \leqslant k \leqslant j\right\}(j \in \mathbf{N}$ does not depend on l) are the only possible positive eigenvalues of $H(l)$. Moreover, the spectrum of $H(l)$ is bounded below; $H(l)$ has void singularly continuous spectrum; and the absolutely continuous and essential spectra of $H(l)$ coincide: they are

$$
\sigma_{\mathrm{ac}}(H(l))=\sigma_{e}(H(l))=[0, \infty) .
$$

Proof. The statement about the positive eigenvalues of $H(l)$ is an immediate consequence of Remark 7.1(a). By Remark 7.1(c), all solutions of $\left(\tau_{l}+\lambda^{2}\right) \phi=0$ $(\lambda>0)$ have only finitely many zeros in $\mathbf{R}_{+}$. This implies that the spectrum of $H(l)$ is bounded below and that $\sigma_{e}(H(l)) \subseteq[0, \infty)$; see [20, Lemma 7.39, p. 1464]. On the other hand, by Corollary 7.5, if $\left[\lambda_{1}, \lambda_{2}\right] \subseteq \mathbf{R}_{j}^{+}$, then $P\left(\left(\lambda_{1}, \lambda_{2}\right)\right) L^{2}\left(\mathbf{R}_{+}\right)$is a nonzero subspace of $L^{2}\left(\mathbf{R}_{+}\right)_{\mathrm{ac}}$. Hence, $\sigma_{\mathrm{ac}}(H(l))$ contains the closure of $\mathbf{R}_{j}^{+}$: i.e., $\sigma_{\mathrm{ac}}(H(l)) \supseteq$ $[0, \infty)$. But $\sigma_{\mathrm{ac}}(H(l)) \subseteq \sigma_{e}(H(l))$ so that (7.3) follows. Finally, the orthogonal complement of $L^{2}\left(\mathbf{R}_{+}\right)_{\text {ac }}$ is contained in $P\left(\mathbf{R} \backslash \mathbf{R}_{j}^{+}\right) L^{2}\left(\mathbf{R}_{+}\right)$which is a subspace spanned by the eigenvalues of $H(l)$ because $\mathbf{R} \backslash \mathbf{R}_{j}^{+}$contains at most countably many points in the spectrum of $H(l)$ (because $\sigma_{e}(H(l))=[0, \infty)$ ). Hence, $H(l)$ has void singularly continuous spectrum. The proof is complete.

8. The spectral representation. We shall now derive a spectral representation (or "eigenfunction expansion") for $H(l)_{\mathrm{ac}}$. In essence Theorems 1.1 and 1.2 are corollaries of this result. We shall call upon the general theory of representations for selfadjoint second order ordinary differential operators.

Suppose, therefore, that $H$ is a selfadjoint restriction of $T_{1}(\tau)$ (in the notation of §2) where $\tau$ is a second order differential expression defined on $\mathbf{R}_{+}$. Let $f_{1} \in L^{2}\left(\mathbf{R}_{+}\right)$ and $A_{2} \in \Sigma(\Sigma$ is the Borel subsets of $\mathbf{R})$. Define the measures on $\Sigma, \mu_{1}=\mu_{f_{1}}$ (notation (7.2)) and $\mu_{2}(A)=\mu_{1}\left(A \cap A_{2}\right), A \in \Sigma$. Form the Hilbert space $\mathcal{H}=$ $L^{2}\left(\mathbf{R}, \Sigma, \mu_{1}\right) \oplus L^{2}\left(\mathbf{R}, \Sigma, \mu_{2}\right)$ and let $P_{n}(n=1$ or 2$)$ be the orthogonal projection of $\mathcal{H}$ onto $L^{2}\left(\mathbf{R}, \Sigma, \mu_{n}\right)$. A unitary operator $U$ from $L^{2}\left(\mathbf{R}_{+}\right)$onto $\mathcal{H}$ is said to be an ordered representation relative to $H$ if, for $n=1$ and 2,

$$
P_{n} U H f(\lambda)=\lambda P_{n} U f(\lambda) \text { for all } f \in L^{2}\left(\mathbf{R}_{+}\right) .
$$

The fundamental result we shall require is as follows. There exists (for some $f_{1} \in L^{2}\left(\mathbf{R}_{+}\right)$and $\left.A_{2} \in \Sigma\right)$ an ordered representation $U$ relative to $H$. Also, $f_{1} \in$ $L^{2}\left(\mathbf{R}_{+}\right)$is maximal in the sense that $\mu_{f} \ll \mu_{1}$ for $f \in L^{2}\left(\mathbf{R}_{+}\right)$(recall $\mu_{1}=\mu_{f_{1}}$ ). Moreover, there exist functions $W_{1}$ and $W_{2}$ in $L_{\text {loc }}^{2}\left(\mathbf{R}_{+} \times \mathbf{R}, \Sigma \otimes \Sigma, m \otimes \mu_{1}\right)(m$ is Lebesgue measure on $\mathbf{R}_{+}$) so that ( $n=1$ or 2 )

$$
\begin{gathered}
P_{n} U F(\lambda)=\lim _{m \in \mathbf{N}} \int_{1 / m}^{m} \overline{W_{n}(r, \lambda)} f(r) d r, \\
U^{-1} g(r)=\lim _{m \in \mathbf{N}} \sum_{n=1,2} \int_{-m}^{m} W_{n}(r, \lambda) P_{n} g(\lambda) d \mu_{1}(\lambda)
\end{gathered}
$$

for all $f \in L^{2}\left(\mathbf{R}_{+}\right)$and $g \in \mathcal{H}$, the limits existing in $\mathcal{H}$ and $L^{2}\left(\mathbf{R}_{+}\right)$, respectively. In addition, $W_{1}$ and $W_{2}$ satisfy:

(1) $(\tau-\lambda) W_{n}(\cdot, \lambda)=0$ for all $\lambda \in \mathbf{R}$ and $n \in\{1,2\}$;

(2) $W_{1}(\cdot, \lambda)$ and $W_{2}(\cdot, \lambda)$ are linearly independent for $\mu_{2}$ almost all $\lambda$ in $\mathbf{R}$; 
(3) $W_{1}(\cdot, \lambda) \neq 0$ for $\mu_{1}$ almost all $\lambda$ in $\mathbf{R}$;

(4) $W_{2}(\cdot, \lambda)=0$ for all $\lambda \in \mathbf{R} \backslash A_{2}$.

For a proof of this result, see [20, Theorem 5.1, p. 1333 and Lemma 6, p. 1209].

Assume now Hypothesis 7.3 and consider the case when $\tau=\tau_{l}$ and $H=H(l)$. We shall show that, in this setting, $W_{1}$ and $W_{2}$ further satisfy: for $\mu_{1}$ almost all $\lambda$ in $\mathbf{R}$

(5) $W_{n}(\cdot, \lambda) \in L^{2}((0,1])$;

(6) $B_{l} W_{n}(\cdot, \lambda)=0\left(B_{l}\right.$ defined by $\left.(2.3)\right)$

for $n=1$ or 2 . Let $\tau_{l}^{\prime}$ be the restriction of $\tau_{l}$ to $(0,1]$ and let $H^{\prime}$ be any selfadjoint operator on $L^{2}((0,1])$ which is a restriction of $T_{1}\left(\tau_{l}^{\prime}\right)$. If we can show that the essential spectrum $\sigma_{e}\left(H^{\prime}\right)$ of $H^{\prime}$ is void then it will follow that (5) and (6) hold by [20, Theorem 13, p. 1402]. To prove $\sigma_{e}\left(H^{\prime}\right)$ is void, recall from Lemma 3.1 that, for all $\lambda>0$, there is a real solution, $\psi(\cdot, \lambda)$ of $\left(\tau_{l}-\lambda^{2}\right) \psi=0$ with only finitely many zeros in $(0,1]$. Therefore, $\sigma_{e}\left(H^{\prime}\right)$ is void by [20, Lemma 39, p. 1464], and hence (5) and (6) hold.

These properties of $W_{1}$ and $W_{2}$ have several consequences. The first, stated in the next lemma, says that $H(l)$ has "spectral multiplicity" one.

Lemma 8.1. Assume Hypothesis 7.3 and the above notation. Then $\mu_{2}=0$.

Proof. By the uniqueness of $\psi$ in Lemma 3.1, $W_{1}(\cdot, \lambda)$ and $W_{2}(\cdot, \lambda)$ are multiples of $\psi(\cdot, \sqrt{\lambda})$ for $\mu_{1}$ almost all $\lambda$. (See (1), (5), and (6) above.) On the other hand, $W_{1}(\cdot, \lambda)$ and $W_{2}(\cdot, \lambda)$ are linearly independent for $\mu_{2}$ almost all $\lambda$, by (2). This apparent contradiction can only be resolved if $\mu_{2}=0$. The proof is complete.

Observe the following immediate consequences of Lemma 8.1: $\mathcal{H}=L^{2}\left(\mathbf{R}, \Sigma, \mu_{1}\right)$; $W_{2}=0 ; P_{2}=0$; and $P_{1}$ is the identity on $\mathcal{H}$. We may now establish the existence of an eigenfunction expansion for $H(l)_{\mathrm{ac}}$.

TheORem 8.2. Assume Hypothesis 7.3 and let $L^{2}\left(\mathbf{R}_{+}\right)_{\mathrm{ac}}$ be the subspace of absolute continuity of $H(l)$ and let $H(l)_{\mathrm{ac}}$ be the absolutely continuous part of $H(l)$. Then there exists a unitary operator $T$ of $L^{2}\left(\mathbf{R}_{+}\right)_{\mathrm{ac}}$ onto $L^{2}\left(\mathbf{R}_{+}\right)$such that

$$
T H(l)_{\mathrm{ac}} f(\lambda)=\lambda T f(\lambda) \text { for all } f \text { in the domain of } H(l)_{\mathrm{ac}} \text {. }
$$

Moreover, there exists $W$ in $L_{\text {loc }}^{2}\left(\mathbf{R}_{+} \times \mathbf{R}_{+}\right)$which belongs to $L^{2}((0,1) \times \Lambda)$ for every compact interval $\Lambda$ in $\mathbf{R}_{j}^{+}$such that

$$
\begin{aligned}
T f(\lambda) & =\lim _{m \in \mathbf{N}} \int_{(1 / m, m)} \overline{W(r, \lambda)} f(r) d r \quad \text { for all } f \in L^{2}\left(\mathbf{R}_{+}\right)_{\mathrm{ac}}, \\
T^{-1} g(r) & =\lim _{m \in \mathbf{N}} \int_{(1 / m, m)} W(r, \lambda) g(\lambda) d \lambda \quad \text { for all } g \in L^{2}\left(\mathbf{R}_{+}\right),
\end{aligned}
$$

the limits existing in $L^{2}\left(\mathbf{R}_{+}\right)$and $L^{2}\left(\mathbf{R}_{+}\right)_{\mathrm{ac}}$, respectively. In addition, for almost all $\lambda>0$

$$
W(r, \lambda)=c(\lambda) \operatorname{Im}(a(\lambda) \phi(r, \sqrt{\lambda})) \text { for all } r>0
$$

where $a: \mathbf{R}_{j}^{+} \rightarrow \mathbf{C} \backslash\{0\}$ is continuously differentiable and both $c$ and $1 / c$ belong to $L_{\mathrm{loc}}^{\infty}\left(\mathbf{R}_{j}^{+}\right)$. 
REMARK. The significance of the equation for $W$ is that $\phi$ can be approximated using (4) of Cond $(\xi)$ and $\xi$ can be approximated from Lemma 6.1 or Remark 6.3. The behavior of $W(r, \lambda)$ as $r \rightarrow \infty$ is, therefore, known.

Proof. We shall begin by constructing $T$. Recall the ordered representation, $U$, its kernel $W_{1}$ and $f_{1} \in L^{2}\left(\mathbf{R}_{+}\right)$introduced in the preceding discussion. (Of course, $W_{2}=0$ by Lemma 8.1.) Recall that $f_{1}$ is maximal in $L^{2}\left(\mathbf{R}_{+}\right)$in the sense that $\mu_{f} \ll \mu_{1}$ for all $f \in L^{2}\left(\mathbf{R}_{+}\right)\left(\mu_{1}=\mu_{f_{1}}\right)$. From Corollaries 7.5 and 7.6 we see that $P\left(\mathbf{R}_{j}^{+}\right)$is the projection of $L^{2}\left(\mathbf{R}_{+}\right)$onto $L^{2}\left(\mathbf{R}_{+}\right)_{\mathrm{ac}}$. This and the maximality of $f_{1}$ imply that $P\left(\mathbf{R}_{j}^{+}\right) f_{1}=g$, say, is maximal in $L^{2}\left(\mathbf{R}_{+}\right)_{\mathrm{ac}}$. In particular, $\mu_{f_{0}} \ll \mu_{g}$ where $f_{0}$ is the element of $L^{2}\left(\mathbf{R}_{+}\right)_{\mathrm{ac}}$ constructed in Corollary 7.5. It follows that $m \ll \mu_{g}$ where $m$ is Lebesgue measure on $\mathbf{R}_{+}$and zero on $(-\infty, 0]$. Hence, $m \sim \mu_{g}$ because $g \in L^{2}\left(\mathbf{R}_{+}\right)_{\mathrm{ac}}$.

From (8.1) we see that

$$
U P\left(\mathbf{R}_{j}^{+}\right) f(\lambda)=\chi_{\mathbf{R}_{j}^{+}}(\lambda) U f(\lambda) \text { for all } f \in L^{2}\left(\mathbf{R}_{+}\right)
$$

so that $U L^{2}\left(\mathbf{R}_{+}\right)_{\text {ac }}$ consists of all $f \in L^{2}\left(\mathbf{R}, \Sigma, \mu_{1}\right)$ which vanish off $\mathbf{R}_{j}^{+}$. Since the Radon-Nikodym derivative of $\mu_{g}$ with respect to $\mu_{f_{1}}$ is $\chi_{\mathbf{R}_{j}^{+}}$(because $g=P\left(\mathbf{R}_{j}^{+}\right) f_{1}$ ), the space $U L^{2}\left(\mathbf{R}_{+}\right)_{\mathrm{ac}}$ can be identified with $L^{2}\left(\mathbf{R}, \Sigma, \mu_{g}\right)$. Therefore if $U^{\prime}$ is the restriction of $U$ to $L^{2}\left(\mathbf{R}_{+}\right)_{\mathrm{ac}}$, then $U^{\prime}$ is a unitary operator from $L^{2}\left(\mathbf{R}_{+}\right)_{\text {ac }}$ onto $L^{2}\left(\mathbf{R}, \Sigma, \mu_{g}\right)$.

Let $\rho$ denote the Radon-Nikodym derivative of $\mu_{g}$ with respect to $m$. Because $\mu_{g} \sim m$ it follows that $S: L^{2}\left(\mathbf{R}, \Sigma, \mu_{g}\right) \rightarrow L^{2}\left(\mathbf{R}_{+}\right)$defined by $S f(\lambda)=(\rho(\lambda))^{1 / 2} f(\lambda)$ is a unitary operator. Defining $T=S U^{\prime}$, we have $T$ is a unitary operator from $L^{2}\left(\mathbf{R}_{+}\right)_{\mathrm{ac}}$ onto $L^{2}\left(\mathbf{R}_{+}\right)$. Because $U$ is an integral operator with kernel $W_{1}, T$ is an integral operator with kernel

$$
W(r, \lambda)=(\rho(\lambda))^{1 / 2} \chi_{\mathbf{R}_{j}^{+}}(\lambda) W_{1}(r, \lambda) .
$$

From this definition it is an easy matter to check that equations (8.3), (8.4a), and (8.4b) for $T$ follow from the corresponding equations (8.1), (8.2a), and (8.2b) for $U$.

It remains to consider the properties of $W$. Recalling the uniqueness of $\psi$ of Lemma 3.1, and the properties of $W_{1}$ listed prior to this theorem, it follows that for almost all $\lambda>0$ there is $c_{1}(\lambda) \in \mathbf{C}$ so that

$$
W(r, \lambda)=c_{1}(\lambda) \psi(r, \sqrt{\lambda}) \text { for all } r>0 .
$$

It is clear that $c_{1}: \mathbf{R}_{+} \rightarrow \mathbf{C}$ is measurable. We shall show that $c_{1}$ and $1 / c_{1}$ belong to $L_{\text {loc }}^{\infty}\left(\mathbf{R}_{j}^{+}\right)$. Then expressing $\psi$ in terms of $\phi$ will complete the proof.

Let $f \in L^{2}\left(\mathbf{R}_{+}\right)$be of compact support and let $\left(\lambda_{1}, \lambda_{2}\right) \subseteq \mathbf{R}_{j}^{+}$. Then, by (8.1) and (8.2a)

$$
\begin{aligned}
\left(P\left(\lambda_{1}, \lambda_{2}\right) f \mid f\right) & =\left(U P\left(\lambda_{1}, \lambda_{2}\right) f U P\left(\lambda_{1}, \lambda_{2}\right) f\right)=\left(S \chi_{\left(\lambda_{1}, \lambda_{2}\right)} U f \mid S \chi_{\left(\lambda_{1}, \lambda_{2}\right)} U f\right) \\
& =\int_{\lambda_{1}}^{\lambda_{2}} \int_{0}^{\infty} \int_{0}^{\infty} \overline{W(s, \lambda)} f(s) W(r, \lambda) \overline{f(r)} d r d s d \lambda .
\end{aligned}
$$

Comparing this expression with (7.1) it follows that: for almost all $\lambda>0$,

$$
|W(r, \lambda)|^{2}=\frac{1}{\pi} \operatorname{Im} K(r, r ; \lambda) \text { for all } r>0 \text {. }
$$


Here we have made use of the continuity of the functions $r \mapsto W(r, \lambda)$ and $K$. Hence by (8.5) and the definition of $K$ (Theorem 7.4), for almost all $\lambda>0$

$$
\pi\left|c_{1}(\lambda)\right|^{2}(\psi(r, \sqrt{\lambda}))^{2}=\psi(r, \sqrt{\lambda}) \operatorname{Im}\{a(\lambda) \phi(r, \sqrt{\lambda})\}
$$

for all $r>0$. (Recall that $\psi(\cdot, \sqrt{\lambda})$ is real-valued.) Now $\psi(\cdot, \sqrt{\lambda})$ as a solution of $\left(\tau_{l}-\lambda\right) \psi=0$ has only discrete zeros, so that for almost all $\lambda>0$

$$
\pi\left|c_{1}(\lambda)\right|^{2} \psi(r, \sqrt{\lambda})=\operatorname{Im}(a(\lambda) \phi(r, \sqrt{\lambda})) \text { for all } r>0
$$

Thus, we see that $\left|c_{1}(\lambda)\right|$ agrees almost everywhere with a continuous function defined on $\mathbf{R}_{j}^{+}$and, in view of property (4) of $\phi$ in $\operatorname{Cond}(\xi)$ this function is nonzero. Hence, $c_{1}$ and $1 / c_{1}$ belong to $L_{\text {loc }}^{\infty}\left(\mathbf{R}_{j}^{+}\right)$. Therefore, by $(8.5), W \in L^{2}\left(\left(0, r_{0}\right) \times \Lambda\right)$ for any $r_{0}>0$ and any compact interval $\Lambda$ in $\mathbf{R}_{j}^{+}$and

$$
W(r, \lambda)=c(\lambda) \operatorname{Im}(a(\lambda) \phi(r, \sqrt{\lambda}))
$$

where $c(\lambda)=\pi^{-1}\left|c_{1}(\lambda)\right|^{-2} c_{1}(\lambda)$. Since $c$ and $1 / c$ clearly belong to $L_{\text {loc }}^{\infty}\left(\mathbf{R}_{j}^{+}\right)$the proof is complete.

9. Proof of Theorem 1.1. Before establishing Theorem 1.1 consider briefly the special case of this theorem when $V(r) \rightarrow 0$ as $r \rightarrow \infty$.

REMARK 9.1. If $V$ satisfies $(V 1)$ and $\left(V 2^{\prime}\right)$ and in addition, $V(r) \rightarrow 0$ as $r \rightarrow \infty$, then $H-H_{0}$ is $H_{0}$ compact ( $H=$ " $-\Delta+V$ "; $H_{0}=$ " $-\Delta$ ") by [46, Theorem 3.1, p. 84]. Hence, the essential spectra of $H$ and $H_{0}$ coincide; i.e., $\sigma_{e}(H)=\sigma_{e}\left(H_{0}\right)=[0, \infty)$ (by [46, Theorem 4.6, p. 16]). Thus, Theorem 1.1 can be extended slightly in the event $V(r) \rightarrow 0$ as $r \rightarrow \infty$.

Establish now Theorem 1.1.

Proof of Theorem 1.1. Let $H(l)$ and $H_{0}(l)(l \in \mathbf{N})$ be the operators defined in $\$ 2$ which correspond to $H$ and $H_{0}$. Clearly, Theorem 8.2 applies to $H_{0}(l)$ as well as $H(l)$ so that the absolutely continuous parts, $H_{0}(l)_{\mathrm{ac}}$ and $H(l)_{\mathrm{ac}}$ are unitarily equivalent to the same operator (see (8.3)) and hence to each other. Therefore, $H_{\mathrm{ac}}$ is unitarily equivalent to $H_{0 \mathrm{ac}}$ (see §2) and because $H_{0 \mathrm{ac}}=H_{0}$ [31, Examples 1.9 and 1.10, p. 520] this proves the required unitary equivalence.

A point $\lambda \in \mathbf{R}$ is an eigenvalue of $H$ if and only if $\lambda$ is an eigenvalue of $H(l)$ for some $l \in \mathbf{N}$. Therefore, Corollary 7.6 implies that $H$ has no positive eigenvalues if $\alpha \neq 1$ but if $\alpha=1$ only finitely many of the points $\frac{1}{4}(k \beta)^{2}, k \in \mathbf{N}$, could be positive eigenvalues of $H$. (Here we have used the fact $j \in \mathbf{N}$ in Corollary 7.6 does not depend on $l$.) It is also clear that $H$ has void singularly continuous spectrum, because the $H(l)$ do (Corollary 7.6). Finally the spectrum of $H$ is bounded below because the spectrum of each $H(l)$ is and because $\left(H\left(l_{1}\right) f \mid f\right) \geqslant\left(H\left(l_{2}\right) f \mid f\right)$ if $\nu\left(l_{1}\right) \geqslant \nu\left(l_{2}\right)$ $\left(\geqslant-\frac{1}{4}\right)$ (in the notation of $(2.1)$ ). The proof is complete.

10. The wave operators. We introduce now the modified wave operators and briefly consider their properties. The Møller wave operators will be realized as a special case of the modified wave operators. 
Let $H=$ " $-\Delta+V$ " and denote by $L^{2}\left(\mathbf{R}^{d}\right)_{\mathrm{ac}}$ and by $H_{\mathrm{ac}}$ the subspace of absolute continuity and the absolutely continuous part of $H$. Let $H_{0}=$ " $-\Delta$ ". Recall the definition of the operator $F(H)$ where $F$ is a Borel measurable function, $F: \mathbf{R} \rightarrow \mathbf{C}$ [20, Chapter XII, §2]. For each $t \in \mathbf{R}$, form the operator $e^{i t H}$ (i.e., take $F(\lambda)=e^{i t \lambda}$ ). Then $\left\{e^{i t H}\right\}_{t \in \mathbf{R}}$ forms a group of unitary operators and, similarly, so does $\left\{e^{i t H_{0}}\right\}_{t \in \mathbf{R}}$. Let $\gamma=\left\{\gamma_{t}\right\}_{t \in \mathbf{R}}$ be a set of Borel measurable functions $\gamma_{t}: \mathbf{R} \rightarrow \mathbf{R}$ and form, for each $t \in \mathbf{R}$, the unitary operators, $\exp \left(-i \gamma_{t}\left(H_{0}\right)\right)$. The modified wave operators, for $\left(H, H_{0}, \gamma\right)$ are said to exist if the limits

$$
\begin{aligned}
& s-\lim _{t \rightarrow \infty} e^{i t H} e^{-i t H_{0}} e^{-i \gamma_{t}\left(H_{0}\right)}=\Omega_{m}^{+}\left(H, H_{0} ; \gamma\right), \\
& \underset{t \rightarrow-\infty}{s-\lim _{t \rightarrow-\infty}} e^{i t H} e^{-i t H_{0}} e^{-i \gamma_{t}\left(H_{0}\right)}=\Omega_{m}^{-}\left(H, H_{0} ; \gamma\right)
\end{aligned}
$$

exist. The notation " $s$-lim" indicates that the limits are to be taken in the strong operator topology. Provided these limits exist the operators $\Omega_{m}^{+}\left(H, H_{0} ; \gamma\right)$ and $\Omega_{m}^{-}\left(H, H_{0} ; \gamma\right)$ so defined are said to be the modified wave operators. They will be referred to frequently as simply $\Omega_{m}^{+}$and $\Omega_{m}^{-}$or $\Omega_{m}^{ \pm}$(where " \pm " can read as either “+” or "-"). As the strong limit of partial isometries $\Omega_{m}^{ \pm}$is itself a partial isometry, it maps $L^{2}\left(\mathbf{R}^{d}\right)$ isometrically onto its range. The operator $\Omega_{m}^{ \pm}$is said to be complete if its range is $L^{2}\left(\mathbf{R}^{d}\right)_{\mathrm{ac}}$.

The Møller wave operators are a special case of the modified wave operators. If $\gamma=\left\{\gamma_{t}\right\}_{t \in \mathbf{R}}$ where for each $t \in \mathbf{R}, \gamma_{t}$ is identically 0 , i.e., $\gamma_{t}=0$ so that $e^{-i \gamma_{t}\left(H_{0}\right)}$ is the identity operator, then the operators $\Omega_{m}^{+}\left(H, H_{0} ; \gamma\right)$ and $\Omega_{m}^{-}\left(H, H_{0} ; \gamma\right)$, provided they exist, are known as the Moller wave operators and are denoted $\Omega^{+}\left(H, H_{0}\right)$ and $\Omega^{-}\left(H, H_{0}\right)$ or simply $\Omega^{+}$and $\Omega^{-}$(or $\left.\Omega^{ \pm}\right)$.

The properties of $\Omega^{ \pm}$are well known [31, Chapter $\left.X, \S 3\right]$. Many of these properties carry over to $\Omega_{m}^{ \pm}$without substantial change. For example, if for all $s \in \mathbf{R}$

$$
\lim _{t \rightarrow \pm \infty} \gamma_{t+s}(\lambda)-\gamma_{t}(\lambda)=0 \text { for almost all } \lambda \text { in } \mathbf{R}
$$

then $\Omega_{m}^{ \pm}$"intertwines" $H$ and $H_{0}$. It is also possible, under suitable conditions on $\gamma$, to attach a physical significance to $\Omega_{m}^{ \pm}$as was done by Dollard in the Coulomb case [18, pp. 31-33]. Because these results will not be required here we shall pursue them no further.

What will be useful is a criterion for when the existence of the modified wave operators implies that of the Moller wave operators. Lemma 10.1 below supplies this criterion.

We shall need the definition of local convergence in measure on a Borel subset $A$ of $\mathbf{R}$. Let $M$ be the set of all complex valued Borel measurable functions defined on $A$ and for each $f_{0} \in M, \varepsilon>0$ and $K$ compact in $A$ define $U_{\varepsilon, K}$ to be the set of all $f \in M$ such that

$$
m\left(\left\{x \in K:\left|f(x)-f_{0}(x)\right|>\varepsilon\right\}\right)<\varepsilon,
$$

where $m$ denotes Lebesgue measure. Then there is a unique topology on $M$ such that all the sets $U_{\varepsilon, K}\left(f_{0}\right)$ form a basis for this topology. When we speak of local convergence in measure on $A$, we refer to convergence in this topology. 
LEMMA 10.1. Let $H$ be any selfadjoint operator in $L^{2}\left(\mathbf{R}^{d}\right)$ and $H_{0}="-\Delta "$. Suppose that $\left\{\gamma^{1}\right\}_{t \in \mathbf{R}}$ and $\left\{\gamma^{2}\right\}_{t \in \mathbf{R}}$ are two sets of real-valued Borel measurable functions and $\Omega_{m}^{ \pm}\left(H, H_{0} ; \gamma^{1}\right)$ and $\Omega_{m}^{ \pm}\left(H, H_{0} ; \gamma^{2}\right)$ exist. Then the range of $\Omega_{m}^{ \pm}\left(H, H_{0} ; \gamma^{1}\right)$ contains that of $\Omega_{m}^{ \pm}\left(H, H_{0} ; \gamma^{2}\right)$ if and only if $\left\{\exp \left(i \gamma_{t}^{1}-\gamma_{t}^{2}\right)\right\}_{t \in \mathbf{R}}$ converges locally in measure on $[0, \infty)$ as $t \rightarrow \pm \infty$ to $\theta_{ \pm}$say. In this event

$$
\Omega_{m}^{ \pm}\left(H, H_{0} ; \gamma^{2}\right)=\Omega_{m}^{ \pm}\left(H, H_{0}, \gamma^{1}\right) \theta_{ \pm}\left(H_{0}\right) \text {. }
$$

For a proof of this result see Hörmander [26, Theorem 3.1].

Returning to the setting of the present result, we suppose that $V$ satisfies $(V 1)$, $(V 2),\left(V 2^{\prime}\right)$ and $(V 3)$ and ask how $\gamma$ should be defined in Theorem 1.2. To answer this, let $\xi \in E$ be the function, with domain $\mathbf{R}_{+} \times \mathbf{C}_{j}$, associated with $V$ by Theorem 3.4. (That is $V$ satisfies $\operatorname{Cond}(\xi)$; note $\xi$ does not depend on $\nu$ in Theorem 3.4.) Define

$$
\zeta(r, \lambda)=\lambda \int_{1}^{r} \xi(\sigma, \lambda) d \sigma \quad \text { for }(r, \lambda) \in \mathbf{R}_{+} \times\left(\mathbf{C}_{j} \cap \mathbf{R}_{+}\right)
$$

so that $\zeta$ is the phase shift of the generalized eigenfunction $\phi$; see (4) of $\operatorname{Cond}(\xi)$. Then $\gamma=\left\{\gamma_{t}\right\}_{t \in \mathbf{R}}$ should be chosen so that it compensates for the phase shift or, more precisely

$$
\gamma_{t}(\lambda)= \begin{cases}-\operatorname{sgn} t \zeta(2|t| \sqrt{\lambda}, \sqrt{\lambda}) & \text { if } \lambda \in \mathbf{R}_{j}^{+} \text {and } t \neq 0 \\ 0 & \text { otherwise }\end{cases}
$$

where

$$
\operatorname{sgn} t= \begin{cases}1 & \text { if } t>0, \\ -1 & \text { if } t<0 .\end{cases}
$$

(Recall that $\mathbf{R}_{j}^{+}=\mathbf{R}_{+} \backslash\left\{\frac{1}{4}(k \beta)^{2}: 1 \leqslant k \leqslant j\right\}=\left\{\lambda>0: \lambda^{2} \in \mathbf{C}_{j}\right\}$.) This definition of $\gamma$ may seem objectionable, because $\xi$ is not uniquely determined by $V$ (Lemma 3.3) but, in view of Lemma 10.1, this is not really surprising. In fact, by Lemma 10.1, if $\xi_{1} \in E$ is another function associated with $V$ by Theorem 3.4 then $\Omega^{ \pm}\left(H, H_{0} ; \gamma\right)$ exists and is complete if and only if $\Omega^{ \pm}\left(H, H_{0} ; \gamma_{1}\right)$ exists and is complete (where $\gamma^{1}$ is the obvious analogue of $\gamma$ ). The choice of $\xi$ is therefore at our disposal. For the proof of Theorem 1.2, the following specification of $\xi$ will be useful.

Lemma 10.2. Suppose $V$ satisfies the hypotheses of Theorem 1.2. Then there is $\delta>\frac{1}{2}$ and $\xi \in E(\delta, \delta)$, with domain $\mathbf{R}_{+} \times \mathbf{C}_{j}$ say so that, for all $\nu \in \mathbf{R}$, the functions $r \rightarrow \nu r^{-2}+V(r)$ satisfy $\operatorname{Cond}(\xi)$ and $\zeta$ defined by (10.1) satisfies: for every compact interval $\Lambda \subseteq \mathbf{C}_{j} \cap \mathbf{R}_{+}, k \in \mathbf{N}$ and $m \geqslant 1$ there is a constant $C>0$ so that for all $(r, \lambda) \in(1, \infty) \times \Lambda$

$$
\begin{gathered}
\left|\frac{d^{k}}{d \lambda^{k}} \zeta(r, \lambda)\right| \leqslant C r^{1-\delta} \\
\left|\frac{d^{k}}{d \lambda^{k}} \zeta^{(m)}(r, \lambda)\right| \leqslant C r^{-m \delta} .
\end{gathered}
$$


Proof. By Theorem 3.4, the functions $\nu r^{-2}+V(r)$ satisfies Cond $(\xi)$ for some $\xi \in E$. If $V$ satisfies (a) of Theorem 1.1 then $\xi \in E\left(\delta_{1}, \delta_{2}\right)$ (by Theorem 3.4) where $\delta_{1}>\frac{1}{2}$ and $\delta_{2}>\frac{1}{2}$ whereas if $V$ satisfies (b) then $\xi \in E\left(2 \delta_{1}, \delta_{2}\right)$ (Corollary 6.2) where $\delta_{1}>\frac{1}{4}$ and $\delta_{2}>\frac{1}{2}$. In either case $\xi \in E(\delta, \delta)$ for some $\delta>\frac{1}{2}$ (Lemma 3.2(iii)).

Verify next (10.3a) and (10.3b). Because $\xi$ belongs to $E(\delta, \delta), \xi$ is a finite sum of functions $h(z) g(r) \cos k \beta r^{\alpha}$ where $h \in D, g \in C(\delta, \delta)$ and $k \in \mathbf{Z}$ and of similar functions where sine replaces cosine. Suppose $\alpha \leqslant \delta$. Then (10.3a) and (10.3b) follow from (1.2). If $\alpha>\delta$ and $k=0$, then again (10.3a) and (10.3b) follow from (1.2). If $\alpha>\delta$ and $k \neq 0$ then the function

$$
(r, z) \rightarrow h(z) \int_{r}^{\infty} g(\sigma) \cos k \beta \sigma^{\alpha} d \sigma
$$

exists and belongs to $A$. By Lemma $3.3, \xi$ can be chosen so that no such terms appear. This verifies (10.3a) and (10.3b) and completes the proof.

11. Proof of Theorem 1.2. The idea of the proof of Theorem 1.2 is to transform the subspaces of absolute continuity of $H(l)$ and $H_{0}(l)$ to $L^{2}\left(\mathbf{R}_{+}\right)$via $T$ and $T_{0}$ (in the notation of $\S 8)$. These transformations take the operators $e^{-i t H(l)}$ and $e^{-i t H_{0}(l)}$ to multiplication by $e^{-i t \lambda}$ (see (8.1)). The existence of the wave operators is, thereby, reduced to showing certain functions converge in $L^{2}\left(\mathbf{R}_{+}\right)$; the completeness is reduced to a triviality. This method of proof is due to Green and Lanford [24] and had been applied by several authors, $[11,12,19,52]$ to the consideration of wave operators. Here it provides an alternative to Hörmander's [26] approach (using Cook's theorem) to the modified wave operators which allows more oscillatory potentials and gives completeness as well.

We shall now prove Theorem 1.2 or more precisely we shall prove the existence and completeness of $\Omega_{m}^{ \pm}\left(H, H_{0} ; \gamma\right)$ where $\gamma$ is defined by (10.2).

Proof OF TheOREM 1.2. In view of the decomposition of $H$ (see §2) it is enough to prove the existence and completeness of $\Omega_{m}^{ \pm}\left(H(l), H_{0}(l) ; \gamma\right)$ for arbitrary $l \in \mathbf{N}$. Here we use the fact $\zeta$ and hence $\gamma$ do not depend on $l$.

We adopt the notation established in $\S 8$. Take $l \in \mathbf{N}$ arbitrary but fixed. Define $F$ to be the set of all $h \in L^{2}\left(\mathbf{R}_{+}\right)$of the form

$$
h(\lambda)=2 i\left(c_{0}(\lambda) a_{0}(\lambda)\right)^{-1} f(\lambda)
$$

where $f \in C_{0}^{\infty}\left(\mathbf{R}_{+}\right)$is real-valued and has support in some compact interval $\Lambda$.

Because $c_{0}, a_{0}, 1 / c_{0}$, and $1 / a_{0}$ all belong to $L_{\mathrm{loc}}^{\infty}\left(\mathbf{R}_{j}^{+} / \mathbf{R}_{j}^{+}\right), F$ is a fundamental subset of $L^{2}\left(\mathbf{R}_{+}\right)$. (A subset of a closed linear space $X$ is said to be a fundamental subspace of $X$ if $X$ is the smallest closed linear space containing it.) Similarly, if we define $F_{+}$to be the set of all $h_{+}$of the form

$$
h_{+}(\lambda)=2 i(c(\lambda) a(\lambda))^{-1} f(\lambda)
$$

( $f$ as before), then $F_{+}$is fundamental in $L^{2}\left(\mathbf{R}_{+}\right)$.

To prove the existence of $\Omega_{m}^{+}\left(=\Omega_{m}^{+}\left(H(l), H_{0}(l) ; \gamma\right)\right)$ it is enough to prove $e^{i t H(l)} e^{-i t H_{0}(l)} e^{-i \gamma_{t}\left(H_{0}(l)\right)} g$ converges as $t \rightarrow \infty$ in $L^{2}\left(\mathbf{R}_{+}\right)$for all $g$ in a fundamental subset of $L^{2}\left(\mathbf{R}_{+}\right)$because all operators are unitary. The appropriate choice of 
fundamental subset here is $T_{0}^{-1}(F)$. In fact, we shall show that, if $h$ is defined by (11.1) for some appropriate $f$, and $h_{+}$is defined by (11.2) for the same $f$, then

$$
e^{-i t H_{0}} e^{-i \gamma_{t}\left(H_{0}(l)\right)} T_{0}^{-1} h-e^{-i t H(l)} T^{-1} h_{+}
$$

converges to 0 in $L^{2}\left(\mathbf{R}_{+}\right)$as $t \rightarrow \infty$. Since $\exp (i t H(l))$ is unitary this will prove the existence of $\Omega_{m}^{+}$and also that $\Omega_{m}^{+} T_{0}^{-1} h=T^{-1} h_{+}$. Because $T^{-1}\left(F_{+}\right)$is fundamental in $L^{2}\left(\mathbf{R}_{+}\right)_{\mathrm{ac}}$ this will also show that $\Omega_{m}^{+}$is complete. (This completeness argument is due to Ben-Artzi and Devinatz [11].)

Let us rewrite the expression (11.3), using (8.4b). Recalling (8.1),

$$
\int_{0}^{\infty} e^{-i t \lambda-i \gamma_{t}(\lambda)} W_{0}(r, \lambda) h(\lambda)-e^{-i t \lambda} W(r, \lambda) h_{+}(\lambda) d \lambda .
$$

We shall show that the function (11.4) of $r$ converges to 0 in $L^{2}\left(\mathbf{R}_{+}\right)$as $t \rightarrow \infty$ and thus prove the existence and completeness of $\Omega_{m}^{+}$. In fact, this will also prove the existence and completeness of $\Omega_{m}^{-}$. To see this take the complex conjugate in (11.4) and replace $t$ by $-t$. Note that $\gamma_{-t}=-\gamma_{t}$. This gives

$$
\begin{aligned}
\int_{0}^{\infty} e^{-i t \lambda-i \gamma_{t}(\lambda)} \overline{W_{0}(r, \lambda) h(\lambda)}-e^{-i t \lambda} \overline{W(r, \lambda) h_{+}(\lambda)} d \lambda \\
=\int_{0}^{\infty} e^{-i t \lambda-i \gamma_{t}(\lambda)} W_{0}(r, \lambda) \tilde{h}(\lambda)-e^{-i t \lambda} W(r, \lambda) h_{-}(\lambda) d(\lambda) \\
=e^{-i t H_{0}(l)-i \gamma_{t}\left(H_{0}(l)\right)} T_{0}^{-1} \tilde{h}-e^{-i t H(l)} T^{-1} h_{-},
\end{aligned}
$$

where

$$
\begin{aligned}
& \tilde{h}(\lambda)=-2 i\left(c_{0}(\lambda) \overline{a_{0}(\lambda)}\right)^{-1} f(\lambda), \\
& h_{-}(\lambda)=-2 i(c(\lambda) \overline{a(\lambda)})^{-1} f(\lambda) .
\end{aligned}
$$

(Here we have used the expression for $W$ obtained in Theorem 8.2 to conclude $\overline{W(r, \lambda) h_{+}(\lambda)}=W(r, \lambda) h(\lambda)$.) Therefore, if the expression (11.4) converges to 0 as $t \rightarrow \infty$, then

$$
e^{-i t H_{0}(l)-i \gamma_{l}\left(H_{0}(l)\right)} T_{0}^{-1} \tilde{h}-e^{-i t H(l)} T^{-1} h_{-}
$$

converges to 0 as $t \rightarrow-\infty$. Since all the $\tilde{h}$ and $h_{-}$form fundamental subsets of $L^{2}\left(\mathbf{R}_{+}\right)$, it will follow that $\Omega_{m}^{-}$exists and is complete.

It remains, therefore, only to show that the expression in (11.4) converges to 0 in $L^{2}\left(\mathbf{R}_{+}\right)$as $t \rightarrow \infty$. We restrict attention to $t>0$ in $(11.4)$ so that $\gamma_{t}(\lambda)=-\zeta(2 t \sqrt{\lambda}, \sqrt{\lambda})$. The proof of convergence involves breaking (11.4) into various components and examining each component. The first breakdown is as follows: for arbitrary $r_{0}>0$ define

$$
\begin{aligned}
& I_{1}(t, r)=\chi_{\left(0, r_{0}\right]}(r) \int_{0}^{\infty} e^{-i t \lambda}\left\{e^{i \xi(2 t \sqrt{\lambda}, \sqrt{\lambda})} W_{0}(r, \lambda) h(\lambda)-W(r, \lambda) h_{+}(\lambda)\right\} d \lambda, \\
& I_{2}(t, r)=\chi_{\left(r_{0}, \infty\right)}(r) \int_{0}^{\infty} e^{-i t \lambda}\left\{e^{i \xi(2 t \sqrt{\lambda}, \sqrt{\lambda})} W_{0}(r, \lambda) h(\lambda)-W(r, \lambda) h_{+}(\lambda)\right\} d \lambda
\end{aligned}
$$

( $\chi$ denotes indicator function). Then $I_{1}+I_{2}$ is the function in (11.4). Now $W$ and $W_{0}$ belong to $L^{2}\left(\left(0, r_{0}\right) \times \Lambda\right)\left(\Lambda \subseteq \mathbf{R}_{j}^{+}\right.$is the support of $h$ and $\left.h_{+}\right)$. Therefore, to prove $\left\|I_{1}(t, \cdot)\right\|$ converges to 0 as $t \rightarrow \infty\left(\|\cdot\|\right.$ denotes the $L^{2}\left(\mathbf{R}_{+}\right)$norm) it suffices 
to prove that, for arbitrary $\psi_{1}$ and $\psi_{2}$ in $C_{0}^{\infty}\left(\mathbf{R}_{+} \times \mathbf{R}_{+}\right)$with support in $\left(0, r_{0}\right) \times \Lambda$,

$$
\int_{0}^{\infty} e^{-i t \lambda}\left\{e^{-i \zeta(2 t \sqrt{\lambda}, \sqrt{\lambda})} \psi_{1}(r, \lambda)-\psi_{2}(r, \lambda)\right\} d \lambda
$$

converges to 0 in $L^{2}\left(\mathbf{R}_{+}\right)$as $t \rightarrow \infty$. This is easily verified by integrating by parts; the factor $e^{-i t \lambda}$ being integrated. See also (10.3a) and (10.3b).

It remains to consider $I_{2}$. Because $r_{0}>0$ was arbitrary in the above argument, it suffices to prove $\left\|I_{2}(t, \cdot)\right\|$ can be made arbitrarily small by choosing $r_{0}$ and $t$ large.

Expand $I_{2}$ as follows. First change variables: set $x=\sqrt{\lambda}$. Then substitute the expression for $W$ from Theorem 8.2 and the corresponding expression for $W_{0}$. (Recall $\xi_{0}=0$ by Corollary 6.5.)

$$
I_{2}=\chi_{\left(r_{0}, \infty\right)}\left(I_{3}+I_{4}+I_{5}+I_{6}+I_{7}\right)
$$

where

$$
\begin{aligned}
& I_{3}(t, r)=\int_{0}^{\infty} e^{-i t x^{2}+i r x}\left\{e^{i \zeta(2 t x, x)}-e^{i \zeta(r, x)}\right\}\left(1+\eta_{1}(r, x)\right) g(x) d x, \\
& I_{4}(t, r)=\int_{0}^{\infty} e^{-i t x^{2}+i r x} e^{i \zeta(2 t x, x)}\left(\eta_{01}(r, x)-\eta_{1}(r, x)\right) g(x) d x, \\
& I_{5}(t, r)=-\int_{0}^{\infty} e^{-i\left(t x^{2}+r x\right)} e^{i \zeta(2 t x, x)} \frac{\overline{a_{0}\left(x^{2}\right)}}{a_{0}\left(x^{2}\right)}\left(1+\overline{\eta_{01}(r, x)}\right) g(x) d x, \\
& I_{6}(t, r)=\int_{0}^{\infty} e^{-i\left(t x^{2}+r x\right)}\left\{e^{-i \zeta(r, x)} \frac{\overline{a\left(x^{2}\right)}}{a\left(x^{2}\right)}\left(1+\overline{\eta_{1}(r, x)}\right)\right\} g(x) d x, \\
& I_{7}(t, r)=\int_{0}^{\infty} e^{-i t x^{2}}\left\{e^{i \zeta(2 t x, x)}\left(e^{i r x} \eta_{02}(r, x)-e^{-i r x} \frac{\overline{a_{0}\left(x^{2}\right)}}{a_{0}\left(x^{2}\right)} \overline{\eta_{02}(r, x)}\right)\right. \\
& \left.\quad-e^{i x r+i \zeta(r, x)} \eta_{2}(r, x)+e^{-i x r-i \zeta(r, x)} \frac{\frac{a\left(x^{2}\right)}{a\left(x^{2}\right)}}{\eta_{2}(r, x)}\right\} g(x) d x,
\end{aligned}
$$

where $g(x)=f\left(x^{2}\right) 2 x$. Define $\lambda_{2}>\lambda_{1}>0$ so that $\left[\lambda_{1}^{2}, \lambda_{2}^{2}\right]=\Lambda$; then $g$ has support in $\left[\lambda_{1}, \lambda_{2}\right]$.

Consider $I_{7}$. Because $\eta_{2}$ and $\eta_{02}$ belong to $L^{2}\left((1, \infty) \times\left[\lambda_{1}, \lambda_{2}\right]\right)$ it is possible to make $\left\|\chi_{\left(r_{0}, \infty\right)} I_{7}(t, \cdot)\right\|$ arbitrarily small for all $t>0$ by simply choosing $r_{0}>0$ suitably large.

Estimate next $\left\|\chi_{\left(r_{0}, \infty\right)} I_{4}(t, \cdot)\right\|$. Because $\eta_{01}$ and $\eta_{1}$ belong to $A, \eta_{01}(r, x)-\eta_{1}(r, x)$ can be written as

$$
\sum_{k=1}^{n} f_{k}(r) h_{k}(x)
$$

where $n \in \mathbf{N}$ and for each $k, h_{k} \in C^{\infty}\left(\mathbf{R}_{+} \backslash A_{j}^{2}\right)$ and $f_{k}(r)=o(1)$ as $r \rightarrow \infty$. We may assume $n=1$. Then $I_{4}(t, r)$ is $f_{1}(r)$ times the Fourier transform, evaluated at $r$ of

$$
e^{-i t x^{2}+i \zeta(2 t x \cdot x)} h_{1}(x) g(x) .
$$


Since the Fourier transform is unitary, for some constant $C>0$,

$$
\left\|\chi_{\left(r_{0}, \infty\right)} I_{4}(t, \cdot)\right\| \leqslant C\left\|h_{1} g\right\| r_{0}^{-\varepsilon} .
$$

Thus, this term can be made arbitrarily small by choosing $r_{0}$ large.

Claim that, when establishing the convergence of the remaining terms, we may assume $\eta_{1}=0=\eta_{01}$. For suppose the convergence has been established in this special case and consider, to be specific, $I_{5}$. The function $\eta_{01}$ may be written in the form (11.5). Certainly, we may assume $n=1$ there. Factor $f_{1}$ out of the integral expression for $I_{5}$ and observe that $h_{1} g \in C_{0}^{\infty}\left(\left[\lambda_{1}, \lambda_{2}\right]\right)$. Since $g$ was arbitrary in $C_{0}^{\infty}\left(\left[\lambda_{1}, \lambda_{2}\right]\right)$ and $f_{1}$ is bounded, the general case $\left(\eta_{01} \neq 0\right)$ follows from the special case. Assume, therefore, that $\eta_{1}=0=\eta_{01}$.

To estimate $I_{5}$, integrate by parts. The factor $\exp \left(-i\left(t x^{2}+r x\right)\right)$ should be integrated. Recalling (10.3a) and (10.3b) and that $a_{0}$ is continuously differentiable, it follows that $\left\|\chi_{\left(r_{0}, \infty\right)} I_{5}(t, \cdot)\right\| \rightarrow 0$ as $t \rightarrow \infty$. Similarly, $\left\|\chi_{\left(r_{0}, \infty\right)} I_{6}(t, \cdot)\right\| \rightarrow 0$ as $t \rightarrow \infty$.

It remains only to consider $I_{3}$. Choose $\psi \in C_{0}^{\infty}(\mathbf{R})$ so that $0 \leqslant \psi(y) \leqslant 1$ for all $y \in \mathbf{R}$ and

$$
\psi(y)= \begin{cases}1 & \text { if }|y| \leqslant 1 \\ 0 & \text { if }|y| \geqslant 2 .\end{cases}
$$

Choose $\varepsilon$ so that $0<\varepsilon<\frac{1}{2} \min \left(\delta-\frac{1}{2}, 1\right)\left(\delta>\frac{1}{2}\right.$ is the constant of Lemma 10.2). Then, making a change of variables, we have

$$
\begin{aligned}
\left\|\chi_{\left(r_{0}, \infty\right)} I_{3}(t, \cdot)\right\| & =\left(\int_{r_{0 / t}}^{\infty} t\left|\int_{0}^{\infty} e^{-i t\left(x^{2}-s x\right)}\left\{e^{i \xi(2 t x, x)}-e^{i \xi(t s, x)}\right\} g(x) d x\right|^{2} d s\right)^{1 / 2} \\
& \leqslant\left\|I_{8}(t, \cdot)\right\|+\left\|I_{9}(t, \cdot)\right\|
\end{aligned}
$$

where

$$
\begin{aligned}
& I_{8}(t, s)=t^{1 / 2} \int_{0}^{\infty} e^{-i t\left(x^{2}-s x\right)}\left\{e^{i \zeta(2 t x, x)}-e^{i \zeta(t s, x)}\right\} \psi\left(t^{1 / 2-\varepsilon}(2 x-s)\right) g(x) d x, \\
& I_{9}(t, s)=t^{1 / 2} \int_{0}^{\infty} e^{-i t\left(x^{2}-s x\right)}\left\{e^{i \zeta(2 t x, x)}-e^{i \zeta(t s, x)}\right\}\left(1-\psi\left(t^{1 / 2-\varepsilon}(2 x-s)\right)\right) g(x) d x .
\end{aligned}
$$

If $\psi\left(t^{1 / 2-\varepsilon}(2 x-s)\right) g(x) \neq 0$, then $\lambda_{1}<s<4 \lambda_{2}$ for all suitably large $t$ and $\left|x-\frac{1}{2} s\right|<t^{-1 / 2+\varepsilon}$. Therefore, for such $s$ and $x$

$$
\left|e^{i \zeta(2 t x, x)}-e^{i \zeta(t s, x)}\right| \leqslant C t^{-\delta+1 / 2+\varepsilon},
$$

for some constant $C>0$, by the mean value theorem and (10.3b). Hence, for some $C>0$,

$$
\left\|I_{8}(t, \cdot)\right\|^{2} \leqslant \int_{\lambda_{1}}^{4 \lambda_{2}} t\left|\int_{|x-s / 2|<t^{-1 / 2+\varepsilon}} C t^{1 / 2+\varepsilon-\delta} d x\right|^{2} d s
$$

so that $\left\|I_{8}(t, \cdot)\right\| \rightarrow 0$ as $t \rightarrow \infty$ by the choice of $\varepsilon$.

Only $I_{9}$ remains. We shall integrate by parts several times; the factor

$$
\exp \left\{i t\left(x^{2}-s x\right)\right\}=\exp \left\{i t(1+s) \frac{x^{2}-s x}{1+s}\right\}
$$


will be integrated. All the derivatives of the function $x \mapsto(1+s)^{-1}\left(x^{2}-s x\right)$ are bounded on $\left[\lambda_{1}, \lambda_{2}\right]$, uniformly in $s$. The first derivative satisfies

$$
\frac{|2 x-s|}{1+s}>C t^{-1 / 2+\varepsilon}
$$

for all $(s, x)$ in the support of $\left(1-\psi\left(t^{1 / 2-\varepsilon}(2 x-s)\right)\right) g(x)$. Combining these remarks and (10.3a) and (10.3b) it is easily checked that $\left\|I_{9}(t, \cdot)\right\| \rightarrow 0$ as $t \rightarrow \infty$. This concludes the proof.

12. Proof of Theorem 1.3. Suppose now that the long range potential, $V_{L}$, is

$$
V_{L}(r)=a r^{-\delta} \sin \beta r^{\alpha} \quad(a \in \mathbf{R} ; \alpha \geqslant 0 ; \beta>0 ; \delta>\min (0,1-\alpha)) .
$$

Applying our results in this situation we shall prove Theorem 1.3. The arguments below naturally extend to any $V_{L}$ in $B\left(\delta_{1}, \delta_{2}\right)$ but establishing Theorem 1.3 will adequately illustrate the techniques.

Proof of Theorem 1.3. The potential $V_{L}$, defined in (12.1), belongs to $B(\delta, 1)$ (in the notation of $\S 1$ ). Therefore, $V_{L}$ satisfies $(L R 1)$ and $(L R 2)$ of assumption (V3); the constants $\delta_{1}$ and $\delta_{2}$ there should be

$$
\delta_{1}=\max (\delta, \delta+\alpha-1) ; \quad \delta_{2}=1 ;
$$

and the functions $V_{L 1}$ and $V_{L 2}$ there can be chosen as: if $\alpha \leqslant 1$, then $V_{L 1}=V_{L}$ and $V_{L .2}=0 ;$ if $\alpha>1$, then

$$
V_{L 1}(r)=\frac{a(1-\delta-\alpha)}{\beta \alpha} r^{-\delta-\alpha} \cos \beta r^{\alpha} ; \quad V_{L 2}=V_{L}-V_{L 1}
$$

Actually, to verify that $V_{L}$ satisfies ( $\left.V 3\right)$, there are many possible choices of $V_{L 1}$ and $V_{L 2}$ but, as we shall soon see, this choice is convenient because

$$
\int_{r}^{\infty} V_{L 2}(\sigma) d \sigma=\frac{\alpha}{\beta \alpha} r^{1-\delta-\alpha} \cos \beta r^{\alpha}, \quad \text { if } \alpha>1
$$

and this last function is in $B\left(\delta_{1}, 1\right)$.

We may now apply Theorem 1.2. Observe that $V_{L, 1}$ is conditionally integrable if either $\alpha>1$ or $\alpha \leqslant 1$ and $\delta+\alpha>1$; in either case

$$
\int_{r}^{\infty} V_{L 1}(\sigma) d \sigma=O\left(r^{-\varepsilon}\right) \text { as } r \rightarrow \infty, \text { for some } \varepsilon>0 .
$$

From this and the value of $\delta_{1}(12.2)$ it follows that the modified wave operators exist and are complete by Theorem 1.2, provided $\delta$ and $\alpha$ satisfy at least one of the conditions (i), (ii), or (iii) of Theorem 1.3.

As yet we have said nothing about the "modification" $\gamma$ of the wave operators, but of course $\gamma=\left\{\gamma_{t}\right\}_{t \in \mathbf{R}}$ is defined by equation (10.2). The existence of the Moller wave operators $\Omega^{ \pm}\left(=\Omega^{ \pm}\left(H, H_{0}\right)\right)$ is determined by the convergence of $\left\{\gamma_{t}\right\}_{t \in \mathbf{R}}$. More precisely, by Lemma $10.1, \Omega^{ \pm}$exists if and only if

$$
\left\{\exp \left(i \gamma_{t}\right)\right\}_{t \in \mathbf{R}} \text { converges locally in measure }
$$

on $\mathbf{R}_{+}\left(=\sigma_{\mathrm{ac}}\left(H_{0}\right)\right)$ as $t \rightarrow \pm \infty$ and in this event $\Omega^{*}$ is complete. It should be remarked that, when applying Lemma 10.1, we have made use of the following general property of $\Omega^{ \pm}$: if $\Omega^{ \pm}$exists, then its range is contained in the subspace 
$L^{2}\left(\mathbf{R}^{d}\right)_{\text {ac }}$ of absolute continuity of $H$ (see [31, Theorem 3.2, p. 531]). (Of course, the range of $\Omega_{m}^{ \pm}$is $L^{2}\left(\mathbf{R}^{d}\right)_{\mathrm{ac}}$ by completeness.)

Therefore to conclude the proof it suffices to show that $\gamma$ satisfies (12.4) if and only if $\delta$ and $\alpha$ satisfy either (iv) or (v) of Theorem 1.3. But it is possible to compute $\gamma$ explicitly from its definition and the expressions obtained for $\xi$ in Remark 6.3. In carrying out this calculation we note that, in the cases $\alpha=1$ and $\alpha>1$, all terms of $\xi$ which are oscillating are conditionally integrable and hence the corresponding terms of $\gamma$ may be eliminated by way of Lemma 10.1. (Similar simplifications can be obtained when $\alpha<1$ by considering cases, but this is not necessary.) We find:

if $\alpha<1$ then

$$
\begin{aligned}
\gamma_{t}(\lambda)=\operatorname{sgn}(t)\{a & (2 \sqrt{\lambda})^{-1} \int_{1}^{2|t| \sqrt{\lambda}} r^{-\delta} \sin \beta r^{\alpha} d r \\
& \left.+a^{2}(2 \sqrt{\lambda})^{-3} \int_{1}^{2|t| \sqrt{\lambda}} r^{-2 \delta} \sin ^{2} \beta r^{\alpha}+x(r, \sqrt{\lambda}) d r\right\}
\end{aligned}
$$

if $\alpha=1$ then

$$
\gamma_{t}(\lambda)=\operatorname{sgn}(t) a^{2}\left(4 \sqrt{\lambda}\left(4 \lambda-\beta^{2}\right)\right)^{-1} \int_{1}^{2|t| \sqrt{\lambda}} r^{-2 \delta}+x(r, \sqrt{\lambda}) d r
$$

if $\alpha>1$ then

$$
\gamma_{t}(\lambda)=\operatorname{sgn}(t) \frac{1}{2}\left(\frac{a}{\beta \alpha}\right)^{2} \sqrt{\lambda} \int_{1}^{2|t| \sqrt{\lambda}} r^{-2(\delta+\alpha-1)}+x(r, \sqrt{\lambda}) d r
$$

where $x(r, \sqrt{\lambda})=O\left(r^{-2 \delta_{1}-\varepsilon}\right)$ as $r \rightarrow \infty(\varepsilon>0)$ for each $\sqrt{\lambda} \in \mathbf{R}_{j}^{+}$. It is now a routine task to check that (12.4) holds if and only if $\delta$ and $\alpha$ satisfy (iv) or (v). This concludes the proof.

REMARK 12.1. Recall the conjectures of Bourgeois and of Mochuzuki and Uchiyama referred to in the Introduction. When $V_{L}(r)=r^{-1 / 2} \sin r$, we see, from Theorem 1.3, that the Moller wave operators do not exist (confirming Bourgeois' conjecture) so that conditional integrability of $V_{L}$ is not, as Mochizuki and Uchiyama suggested a sufficient condition for the Moller wave operators to exist. It is, however, necessary at least in the present context, and if we further suppose that $V_{L}=V_{L 1}+V_{L 2}$ where $V_{L 1}$ and $\int_{r}^{\infty} V_{L 2}(\sigma) d \sigma$ belong to $L^{2}((1, \infty))$ we get the desired necessary and sufficient condition. It seems likely that, even for more general central potentials than those considered here, this should be the appropriate condition. (Say if $V$ satisfies $(V 1),(V 2)$ and $\left(V 2^{\prime}\right)$.) What the condition should be when $V$ is not central is not clear.

ACKnowledgement. It is a pleasure to thank Professor A. Devinatz for many helpful discussions and suggestions. I thank also Professor R. S. Phillips and Stanford University for their hospitality during the preparation of this paper.

\section{REFERENCES}

1. S. Agmon, Spectral properties of Schrödinger operators and scattering theory, Ann. Scuola Norm. Sup. Pisa 2 (1975), 151-218.

2. V. De Alfaro and T. Regge, Potential scattering, Wiley, New York, 1965

3. W. O. Amrein and D. B. Pearson, The scattering matrix for rapidly oscillating potentials, J. Phys. A 13 (1980), 1259-1264. 
4. M. Arai, Eigenfunction expansions associated with Schrödinger operators with long-range potentials, Publ. Res. Inst. Math. Sci. 16 (1980), 35-39.

5. F. V. Atkinson, The asymptotic solution of second-order differential equations, Ann. Mat. Pura Appl. 37 (1954), 347-378

6. M. L. Baetemann and K. Chadan, Scattering theory with highly singular, oscillating potentials, Ann. Inst. H. Poincaré Sect. A 24 (1976), 1-16.

7. M. Ben-Artzi, Eigenfunction expansion for a class of differential operators, J. Math. Anal. Appl. 69 (1979), 304-314.

8. On the absolute continuity of Schrödinger operators with spherically symmetric long range potentials. I, J. Differential Equations 38 (1980), 41-50.

9. On the absolute continuity of Schrödinger operators with spherically symmetric long range potentials. II, J. Differential Equations 38 (1980), 51-60.

10. Spectral properties of linear ordinary differential operators with slowly decreasing coefficients, J. Math. Anal. Appl. 68 (1979), 68-91.

11. M. Ben-Artzi and A. Devinatz, Spectral and scattering theory for the adiabatic oscillator and related potentials, J. Math. Phys. 20 (1979), 594-607.

12. B. Bourgeois, On scattering theory for oscillatory potentials of slow decay, Ann. Physics 121 (1979), 415-431.

13. E. A. Coddington and N. Levinson, Theory of ordinary differential equations, McGraw-Hill, New York, 1955, p. 92.

14. M. Combescure, Spectral and scattering theory for a class of strongly oscillating potentials, Comm. Math. Phys. 73 (1980), 43-62.

15. M. Combescure and J. Ginibre, Spectral and scattering theory for the Schrödinger operator with strongly oscillating potentials, Ann. Inst. H. Poincaré Sect. A 24 (1976), 17-29.

16. A.Devinatz, The existence of wave operators for oscillating potentials, J. Math. Phys. 21 (1980), 2406-2411.

17. J. D. Dollard, Asymptotic convergence and the Coulomb interactions, J. Math. Phys. 5 (1964), 729-738.

18. Quantum-mechanical scattering theory for short-range and Coulomb interactions, Rocky Mountain J. Math. 1 (1971), 5-88.

19. J. D. Dollard and C. N. Friedman, Existence of Moller wave operators for $V(r)=\lambda \sin \left(\mu r^{\alpha}\right) / r^{\beta}$, Ann. Physics 111 (1978), 251-266.

20. N. Dunford and J. T. Schwartz, Linear operators. Part II, Interscience, New York, 1963.

21. V. Enss, Asymptotic completeness for quantum mechanical potential scattering. I. Short range potentials, Comm. Math. Phys. 61 (1978), 285-291.

22. Asymptotic completeness for quantum mechanical potential scattering. II. Singular and long range potentials, Ann. Physics 119 (1979), 117-132.

23. J. Ginibre, La méthode 'dépendant du temps' dans le problème de la complétude asymptotique, preprint.

24. T. A. Green and O. E. Lanford, III, Rigourous derivation of the phase shift formula for the Hilbert space scattering operator of a single particle, J. Math. Phys. 1 (1960), 139-148.

25. W. A. Harris, Jr. and D. A. Lutz, Asymptotic integration of adiabatic oscillators, J. Math. Anal. Appl. 51 (1975), 76-93.

26. L. Hörmander, The existence of wave operators in scattering theory, Math. Z. 146 (1976), 69-71.

27. T. Ikebe and H. Isozaki, Completeness of modified wave operators for long-range potentials, Publ. Res. Inst. Math. Sci. 15 (1979), 679-718.

28. H. Isozaki, Eikonal equations and spectral representations for long range Schrödinger Hamiltonians, J. Math. Kyoto Univ. 20 (1980), 243-261.

29. A. R. Its and V. B. Matveev, Co-ordinate asymptotics for the Schrödinger equation with a rapidly oscillating potential, J. Soviet Math. 11 (1979), 442-444.

30. K. Jorgens and J. Weidmann, Spectral properties of Hamiltonian operators, Springer-Verlag, Berlin, 1973.

31. T. Kato, Perturbation theory for linear operators, 2nd ed., Springer-Verlag, Berlin, 1976.

32. H. Kitada, Scattering theory for Schrödinger operators with long-range potentials. I. Abstract theory, J. Math. Soc. Japan 29 (1977), 665-691.

33. Scattering theory for Schrödinger operators with long-range potentials. II. Spectral and scattering theory, J. Math. Soc. Japan 30 (1978), 603-632. 
34. H. Kitada and K. Yajima, A scattering theory for time-dependent long-range potentials, preprint.

35. V. B. Matveev, Wave operators and positive eigenvalues for a Schrödinger equation with oscillating potential, Theoret. and Math. Phys. 15 (1973), 574-583.

36. V. B. Matveev and M. M. Skriganov, Wave operators for the Schrödinger equation with rapidly oscillating potential, Dokl. Akad. Nauk SSSR 13 (1972), 185-188.

37. K. Mochizuki and J. Uchiyama, Radiation conditions and spectral theory for 2-body Schrödinger operators with 'oscillating' long-range potentials. I, J. Math. Kyoto Univ. 18 (1978), 377-408.

38. __ Radiation conditions and spectral theory for 2-body Schrödingerr operators with 'oscillating' long-range potentials. II, J. Math. Kyoto Univ. 19 (1979), 47-70.

39. Time dependent representations of the stationary wave operators for 'oscillating' long-range potentials, preprint.

40. J. von Neumann and E. Wigner, Über merkwürdige diskrete Eigenwerte, Z. Phys. 30 (1929), 465-467.

41. D. B. Pearson, Scattering theory for a class of oscillating potentials, Helv. Phys. Acta 52 (1979), $541-554$.

42. P. A. Perry, Propagation of states in dilation analytic potentials and asymptotic completeness, Comm. Math. Phys. 81 (1981), 243-259.

43. M. Reed and B. Simon, Methods of modern mathematical physics, vol. II, Academic Press, New York, 1975.

44. , Methods of modern mathematical physics, vol. III, Academic Press, New York, 1979.

45. Y. Saito, Spectral representations for Schrödinger operators with long range potentials, Springer-Verlag, Berlin, 1979.

46. M. Schechter, Spectra of partial differential operators, North-Holland, Amsterdam, 1971.

47. _ Scattering theory for elliptic operators of arbitrary order, Comment. Math. Helv. 49 (1974), 84-113.

48. B. Simon, Phase space analysis of simple scattering systems: Extensions of some work of Enss, Duke Math. J. 46 (1979), 119-168.

49. M. M. Skriganov, On the spectrum of the Schrödinger operator with rapidly oscillating potential, Trudy Mat. Inst. Steklov 125 (1973), 177-185.

50. The eigenvalues of the Schrödinger operator situated on the continuous spectrum, J. Soviet Math. 8 (1977), 464-467.

51. H. Tamuro, The principle of limiting absorption for uniformly propagative systems with perturbations of long-range class, Nagoya Math. J. 82 (1981), 141-174.

52. M. Wolfe, Asymptotic behavior of solutions of the radial Schroedinger equation and applications to long-range potential scattering, Dissertation, University of Texas at Austin, 1978.

53. D. Yafaev, On the proof of Enss of asymptotic completeness in potential scattering, preprint, Steklov Institute, Leningrad.

54. B. Bourgeois, Quantum mechanical scattering for potentials of the form $\sin r / r^{\beta}, \frac{1}{3}<\beta \leqslant \frac{1}{2}$, J. Math. Phys. 23 (1982), 790-797.

\section{Department of Mathematics, Stanford University, Stanford, California 94305}

Current address: Department of Mathematics, University of British Columbia, Vancouver, British Columbia, V6T 1W5, Canada 